\title{
On a class of linear functional equations without range condition
}

\author{
Eszter Gselmann, Gergely Kiss, and Csaba Vincze \\ Dedicated to the 95th birthday of Professor János Aczél.
}

\begin{abstract}
The main purpose of this work is to provide the general solutions of a class of linear functional equations. Let $n \geq 2$ be an arbitrarily fixed integer, let further $X$ and $Y$ be linear spaces over the field $\mathbb{K}$ and let $\alpha_{i}, \beta_{i} \in \mathbb{K}, i=1, \ldots, n$ be arbitrarily fixed constants. We will describe all those functions $f, f_{i, j}: X \times Y \rightarrow \mathbb{K}, i, j=1, \ldots, n$ that fulfill the functional
\end{abstract} equation

$$
\begin{gathered}
f\left(\sum_{i=1}^{n} \alpha_{i} x_{i}, \sum_{i=1}^{n} \beta_{i} y_{i}\right)=\sum_{i, j=1}^{n} f_{i, j}\left(x_{i}, y_{j}\right) \\
\left(x_{i} \in X, y_{i} \in Y, i=1, \ldots, n\right) .
\end{gathered}
$$

Additionally, necessary and sufficient conditions will also be given that guarantee the solutions to be non-trivial.

Mathematics Subject Classification. Primary 39B52; Secondary 39B22.

Keywords. Linear functional equation, Polynomial function, Additive function, Bi-additive function, Non-trivial solution.

\section{Introduction}

As János Aczél wrote in his famous and pioneering monograph [1]: 'Functional equations have a long history and occur almost everywhere. Their influence and applications can be felt in every field, and all fields benefit from their contact, use, and technique.' Almost the same can be said about the class of

E. Gselmann was supported by the EFOP-3.6.1-16-2016-00022 project. The project is cofinanced by the European Union and the European Social Fund. G. Kiss was supported by the Hungarian Scientific Research Fund (OTKA) Grant K 124749. Cs. Vincze was supported by EFOP 3.6.2-16-2017-00015. The project is co-financed by the European Union and the European Social Fund. 
linear functional equations. This area is one of the most investigated topic in this field, several authors have studied this class, see e.g. [2-10,14,18-20].

The main purpose of this paper is to describe the general solutions of a class of linear functional equations. More precisely, we are interested in the following problem. Let $n \geq 2$ be an arbitrarily fixed integer, let further $X$ and $Y$ be linear spaces over the field $\mathbb{K}$ and let $\alpha_{i}, \beta_{i} \in \mathbb{K}, i=1, \ldots, n$ be arbitrarily fixed constants. Assume further that for functions $f, f_{i, j}: X \times Y \rightarrow \mathbb{K}, i, j=1, \ldots, n$, the functional equation

$$
f\left(\sum_{i=1}^{n} \alpha_{i} x_{i}, \sum_{i=1}^{n} \beta_{i} y_{i}\right)=\sum_{i, j=1}^{n} f_{i, j}\left(x_{i}, y_{j}\right) \quad\left(x_{i} \in X, y_{i} \in Y, i=1, \ldots, n\right)
$$

is fulfilled.

This equation belongs to the class of linear functional equations, which was thoroughly investigated by L. Székelyhidi in [15-17]. For the sake of completeness, here we briefly recall the main results from Székelyhidi [15].

Definition 1. If $G, S$ are groups and $n$ is a positive integer, then a function $A: G^{n} \rightarrow S$ is said to be $n$-additive if it is a homomorphism in each variable. Let $F: G^{n} \rightarrow S$ be a function, then the function $\varphi: G \rightarrow S$ defined by

$$
\varphi(x)=F(x, \ldots, x) \quad(x \in G)
$$

is said to be the diagonal of $F$ and it is denoted by $\operatorname{diag}(F)$. Further, let

$$
A_{k}(x, y)=A(\underbrace{x, \ldots, x}_{k \text { times }}, \underbrace{y, \ldots, y}_{n-k \text { times }}) \quad(x, y \in G) .
$$

Remark. Let $G, S$ be groups, $n$ be a positive integer and $A: G^{n} \rightarrow S$ be an $n$-additive function. Then for all $k \in \mathbb{Z}$ and arbitrary $i \in\{1, \ldots, n\}$ we have

$$
\begin{aligned}
A & \left(x_{1}, \ldots, x_{i-1}, k x_{i}, x_{i+1}, \ldots, x_{n}\right) \\
= & k A\left(x_{1}, \ldots, x_{i-1}, x_{i}, x_{i+1}, \ldots, x_{n}\right) \\
& \left(x_{1}, \ldots, x_{n} \in G\right) .
\end{aligned}
$$

For a function $f, \operatorname{rng}(f)$ denotes the range of $f$.

Definition 2. Let $G, S$ be Abelian groups, let $n$ be a non-negative integer. A function $f: G \rightarrow S$ is said to be of degree $n$, if there exist functions $f_{i}: G \rightarrow S$ and homomorphisms $\varphi_{i}, \psi_{i}: G \rightarrow G$ such that

$$
\operatorname{rng}\left(\varphi_{i}\right) \subset \operatorname{rng}\left(\psi_{i}\right) \quad(i=1,2, \ldots, n+1)
$$

and the functional equation

$$
f(x)+\sum_{i=1}^{n+1} f_{i}\left(\varphi_{i}(x)+\psi_{i}(y)\right)=0 \quad(x, y \in G)
$$

holds. 
Definition 3. Let $G, S$ be Abelian groups, let $n$ be a non-negative integer. A function $f: G \rightarrow S$ is called a (generalized) polynomial of degree $n$, if for all $k=0,1, \ldots, n$ there exists a $k$-additive mapping $A_{k}: G^{k} \rightarrow S$ such that

$$
f=\sum_{k=0}^{n} \operatorname{diag}\left(A_{k}\right)
$$

where 0-additive functions are understood to be constant functions.

Theorem 1. (Theorem 3.6 of [15]) Let $G, S$ be Abelian groups and suppose that $G$ is divisible. Let $n$ be a non-negative integer. A function $f: G \rightarrow S$ is of degree $n$ if and only if it is a polynomial of degree $n$.

Theorem 2. (Theorem 3.9 of [15]) Let $G, S$ be Abelian groups and suppose that $G$ is divisible and $S$ is torsion free. Let $n \in \mathbb{N}$ be a non-negative integer and let $\varphi_{i}, \psi_{i}$ be homomorphisms of $G$ onto itself such that

$$
\operatorname{rng}\left(\psi_{j} \circ \psi_{i}^{-1}-\varphi_{j} \circ \varphi_{i}^{-1}\right)=G \quad(i \neq j, i, j=1, \ldots, n+1) .
$$

Functions $f_{i}: G \rightarrow S(i=0,1, \ldots, n+1)$ satisfy the functional equation

$$
f_{0}(x)+\sum_{i=1}^{n+1} f_{i}\left(\varphi_{i}(x)+\psi_{i}(y)\right)=0 \quad(x, y \in G)
$$

if and only if for all $k=0,1, \ldots, n$ and $i=0,1, \ldots, n+1$ there exist symmetric $k$-additive functions $A_{k}^{(i)}: G^{k} \rightarrow S$ such that

$$
f_{i}=\sum_{k=0}^{n} \operatorname{diag}\left(A_{k}^{(i)}\right) \quad(i=0,1, \ldots, n+1)
$$

and the equations

$$
A_{k, j}^{(0)}(x, 0)+\sum_{i=1}^{n+1} A_{k, j}^{(i)}\left(\varphi_{i}(x), \psi_{i}(y)\right)=0 \quad(x, y \in G)
$$

hold for all $j=0,1, \ldots, n$ and $k=j, j+1, \ldots, n$.

Observe that Eq. (1) can be reduced to the form (2). Indeed, suppose that $n=2$ (or substitute zero in place of the variables except a distinguished pair) and consider the following family of homomorphisms

$$
\varphi_{\alpha, \beta}(x, y)=\left(\begin{array}{ll}
\alpha & 0 \\
0 & \beta
\end{array}\right) \cdot\left(\begin{array}{l}
x \\
y
\end{array}\right) \quad(x \in X, y \in Y, \alpha, \beta \in \mathbb{K}) .
$$

With these notations (1) can be re-written as

$$
\begin{aligned}
& f\left(\varphi_{\alpha_{1}, \beta_{1}}(\mathbf{u})+\varphi_{\alpha_{2}, \beta_{2}}(\mathbf{v})\right)=f_{1,1}\left(\varphi_{1,1}(\mathbf{u})\right. \\
& \left.+\varphi_{0,0}(\mathbf{v})\right)+f_{1,2}\left(\varphi_{1,0}(\mathbf{u})+\varphi_{0,1}(\mathbf{v})\right) \\
& +f_{2,1}\left(\varphi_{0,1}(\mathbf{u})+\varphi_{1,0}(\mathbf{v})\right)+f_{2,2}\left(\varphi_{0,0}(\mathbf{u})+\varphi_{1,1}(\mathbf{v})\right) \\
& \quad(\mathbf{u}, \mathbf{v} \in X \times Y) .
\end{aligned}
$$


At the same time (as it can be seen in the following subsection), we cannot state that the functions involved are polynomials. This is because of the fact that the homomorphisms $\varphi_{\alpha, \beta}$ defined above in general do not fulfill the range condition $\left(\mathscr{R}_{1}\right)$, nor range condition $\left(\mathscr{R}_{2}\right)$. What is more, they are injective if and only if $\alpha, \beta \neq 0$ and in such a situation $\varphi_{\alpha, \beta}^{-1}=\varphi_{\alpha^{-1}, \beta^{-1}}$. Notice that Eq. (1) involves the projections $\varphi_{1,0}, \varphi_{0,1}$ and $\varphi_{0,0}$. None of these are injective. This shows that Theorems 1 and 2 cannot be applied in our situation.

\section{Special cases of the original equation}

\subsection{The one-variable sub-case}

In this sub-case let $n \in \mathbb{N}, n \geq 2$ be arbitrarily fixed, $X$ be a linear space over the field $\mathbb{K}$ and suppose that for functions $f, f_{1}, \ldots, f_{n}: X \rightarrow \mathbb{K}$ the functional equation

$$
f\left(\sum_{i=1}^{n} \alpha_{i} x_{i}\right)=\sum_{i=1}^{n} f_{i}\left(x_{i}\right) \quad\left(x_{1}, \ldots, x_{n} \in X\right)
$$

holds with certain constants $\alpha_{1}, \ldots, \alpha_{n} \in \mathbb{K}$.

Observe that without loss of generality

$$
f(0)=f_{1}(0)=\ldots=f_{n}(0)=0
$$

can be assumed. Otherwise we consider the functions

$$
\begin{aligned}
\widetilde{f}(x) & =f(x)-f(0) \\
\widetilde{f}_{1}(x) & =f_{1}(x)-f_{1}(0) \quad(x \in X) . \\
& \vdots \\
\widetilde{f_{n}}(x) & =f_{n}(x)-f_{n}(0)
\end{aligned}
$$

They clearly vanish at zero and they also fulfill the above functional equation. Therefore from now on we always suppose that $(*)$ holds.

As we will see, the solutions of Eq. (3) heavily depend on whether or not there are zeros among the parameters $\alpha_{1}, \ldots, \alpha_{n}$. We may (and also do) assume that these parameters are arranged in the following way: there exists a nonnegative integer $k \leq n$ such that $\alpha_{i} \neq 0$ for $i=1, \ldots, k$, but $\alpha_{i}=0$ for all $i=k+1, \ldots, n$.

Proposition 1. Let $n \in \mathbb{N}, n \geq 2$ be arbitrarily fixed, $X$ be a linear space over the field $\mathbb{K}$ and suppose that for functions $f, f_{1}, \ldots, f_{n}: X \rightarrow \mathbb{K}$ the functional Eq. (3) holds with certain constants $\alpha_{1}, \ldots, \alpha_{n} \in \mathbb{K}$ and assume that $(*)$ is also satisfied. Suppose further that $\alpha_{i} \neq 0$ for $i=1, \ldots, k$, but $\alpha_{i}=0$ for all $i=k+1, \ldots, n$. Then

(i) in case $k=0$, all the functions $f_{1}, \ldots, f_{n}$ are identically zero and $f: X \rightarrow$ $\mathbb{K}$ is any function fulfilling $f(0)=0$, 
(ii) in case $k=1$, all the functions $f_{2}, \ldots, f_{n}$ are identically zero and $f, f_{1}$ : $X \rightarrow \mathbb{K}$ are any functions vanishing at zero and fulfilling

$$
f\left(\alpha_{1} x\right)=f_{1}(x) \quad(x \in X),
$$

(iii) otherwise, there exists an additive function $\chi: X \rightarrow \mathbb{K}$ such that

$$
f(x)=\chi(x) \quad \text { and } \quad f_{i}(x)=\chi\left(\alpha_{i} x\right) \quad \text { for } \quad i=1, \ldots, k
$$

and the functions $f_{k+1}, \ldots, f_{n}$ are identically zero.

Conversely, the mappings $f, f_{1}, \ldots, f_{n}: X \rightarrow \mathbb{K}$ vanish at zero and they also fulfill (3).

Proof. In case $k=0$ Eq. (3) reduces to

$$
\sum_{i=1}^{n} f_{i}\left(x_{i}\right)=0 \quad\left(x_{1}, \ldots, x_{n} \in X\right) .
$$

Since we have independent variables, this immediately yields that the involved functions have to be constant functions. In view of $(*)$ this means that they have to be identically zero and the only information we get for the function $f$ is that $f(0)=0$.

In case $k \geq 1$, our equation can be written as

$$
f\left(\sum_{i=1}^{k} \alpha_{i} x_{i}\right)=\sum_{j=1}^{n} f_{j}\left(x_{j}\right) \quad\left(x_{1}, \ldots, x_{n} \in X\right) .
$$

With the substitution

$$
x_{1}=\ldots=x_{k}=0
$$

we obtain that

$$
0=\sum_{j=k+1}^{n} f_{j}\left(x_{j}\right) \quad\left(x_{k+1}, \ldots, x_{n} \in X\right),
$$

which (similarly as above) yields that the functions $f_{k+1}, \ldots, f_{n}$ are identically zero. Using this, the functions $f, f_{1}, \ldots, f_{k}$ fulfill

$$
f\left(\sum_{i=1}^{k} \alpha_{i} x_{i}\right)=\sum_{i=1}^{k} f_{i}\left(x_{i}\right) \quad\left(x_{1}, \ldots, x_{k} \in X\right) .
$$

If $k=1$, this is nothing but

$$
f\left(\alpha_{1} x\right)=f_{1}(x) \quad(x \in X),
$$

showing that in this case there is nothing to prove.

Assume that $k \geq 2$ and let $i, j \in\{1, \ldots, k\}$ be different integers. Then Eq. (4) with $x_{l}=0$ for $l \in\{1, \ldots, k\} \backslash\{i, j\}$ is

$$
f\left(\alpha_{i} x_{i}+\alpha_{j} x_{j}\right)=f_{i}\left(x_{i}\right)+f_{j}\left(x_{j}\right) \quad\left(x_{i}, x_{j} \in X\right),
$$


which, after introducing the functions

$$
\widetilde{f}_{l}(x)=f_{l}\left(\frac{x}{\alpha_{l}}\right) \quad(x \in X, l=1, \ldots, k)
$$

can be reduced to the system of Pexider equations

$$
f\left(x_{i}+x_{j}\right)=\tilde{f}_{i}\left(x_{i}\right)+\tilde{f}_{j}\left(x_{j}\right) \quad\left(x_{i}, x_{j} \in X, i, j \in\{1, \ldots, k\}, i \neq j\right) .
$$

This means that there exists an additive function $\chi: X \rightarrow \mathbb{K}$ such that

$$
f(x)=\chi(x) \text { and } f_{i}(x)=\chi\left(\alpha_{i} x\right) \text { for } i=1, \ldots, k .
$$

\section{The two-variable case with $n=2$}

In this section we will focus on the functional equation

$$
\begin{aligned}
f & \left(\alpha_{1} x_{1}+\alpha_{2} x_{2}, \beta_{1} y_{1}+\beta_{2} y_{2}\right) \\
= & f_{1,1}\left(x_{1}, y_{1}\right)+f_{1,2}\left(x_{1}, y_{2}\right)+f_{2,1}\left(x_{2}, y_{1}\right)+f_{2,2}\left(x_{2}, y_{2}\right) \\
& \left(x_{1}, x_{2} \in X, y_{1}, y_{2} \in Y\right),
\end{aligned}
$$

where $f, f_{1,1}, f_{1,2}, f_{2,1}, f_{2,2}: X \times Y \rightarrow \mathbb{K}$ denote the unknown functions and $\alpha_{1}, \alpha_{2}, \beta_{1}, \beta_{2} \in \mathbb{K}$ are given constants.

Observe that without loss of generality

$$
f(0,0)=f_{i, j}(0,0)=0
$$

can be supposed. Otherwise we consider the functions

$$
\begin{aligned}
\tilde{f}(x, y) & =f(x, y)-f(0,0) \\
\widetilde{f_{i, j}}(x, y) & =f_{i, j}(x, y)-f_{i, j}(0,0)
\end{aligned} \quad(x \in X, y \in Y) .
$$

They clearly vanish at the point $(0,0)$ and they also fulfill the same functional equation. Similarly as before, from now on we always suppose that all the involved functions vanish at the point $(0,0)$.

This section will be divided into two parts. In the first one, we will consider the so-called degenerate cases, where at least one of the parameters $\alpha_{1}, \alpha_{2}, \beta_{1}, \beta_{2}$ is zero. After that the non-degenerate case will follow, that is, when none of the above parameters are zero.

\subsection{Degenerate cases}

3.1.1. The homogeneous case $\boldsymbol{\alpha}_{1}=\boldsymbol{\alpha}_{\mathbf{2}}=\boldsymbol{\beta}_{\mathbf{1}}=\boldsymbol{\beta}_{\mathbf{2}}=\mathbf{0}$. In case $\alpha_{1}=\alpha_{2}=$ $\beta_{1}=\beta_{2}=0$ Eq. (5) reduces to

$$
\begin{aligned}
& f_{1,1}\left(x_{1}, y_{1}\right)+f_{1,2}\left(x_{1}, y_{2}\right)+f_{2,1}\left(x_{2}, y_{1}\right)+f_{2,2}\left(x_{2}, y_{2}\right)=0 \\
& \quad\left(x_{1}, x_{2} \in X, y_{1}, y_{2} \in Y\right) .
\end{aligned}
$$


Proposition 2. Let $X$ and $Y$ be linear spaces over the field $\mathbb{K}$ and $f_{1,1}, f_{1,2}$, $f_{2,1}, f_{2,2}: X \times Y \rightarrow \mathbb{K}$ be functions such that

$$
\begin{aligned}
& f_{1,1}\left(x_{1}, y_{1}\right)+f_{1,2}\left(x_{1}, y_{2}\right)+f_{2,1}\left(x_{2}, y_{1}\right)+f_{2,2}\left(x_{2}, y_{2}\right)=0 \\
& \quad\left(x_{1}, x_{2} \in X, y_{1}, y_{2} \in Y\right) .
\end{aligned}
$$

Then and only then for all $i, j=1,2$ there exist functions $\chi_{i, j}: X \rightarrow \mathbb{K}$ and $\zeta_{i, j}: Y \rightarrow \mathbb{K}$ vanishing at 0 , such that

$$
f_{i, j}(x, z)=\chi_{i, j}(x)+\zeta_{i, j}(z) \quad(x \in X, z \in Y, i, j=1,2)
$$

as well as

$$
\begin{aligned}
& \chi_{1,2}(x)+\chi_{1,1}(x)=0 \\
& \chi_{2,2}(x)+\chi_{2,1}(x)=0 \\
& \zeta_{2,1}(z)+\zeta_{1,1}(z)=0 \\
& \zeta_{2,2}(z)+\zeta_{1,2}(z)=0 \\
& (x \in X, z \in Y) \text {. }
\end{aligned}
$$

Proof. For $i, j \in\{1,2\}$ let us define the functions $\chi_{i, j}: X \rightarrow \mathbb{K}$ and $\zeta_{i, j}: Y \rightarrow$ $\mathbb{K}$ through

$$
\chi_{i, j}(x)=f_{i, j}(x, 0) \quad \text { and } \quad \zeta_{i, j}(z)=f_{i, j}(0, z) \quad(x \in X, z \in Y) .
$$

With the notation

$$
\begin{aligned}
& E\left(x_{1}, x_{2}, y_{1}, y_{2}\right) \\
& =f_{1,1}\left(x_{1}, y_{1}\right)+f_{1,2}\left(x_{1}, y_{2}\right)+f_{2,1}\left(x_{2}, y_{1}\right)+f_{2,2}\left(x_{2}, y_{2}\right) \\
& \quad\left(x_{1}, x_{2} \in X, y_{1}, y_{2} \in Y\right)
\end{aligned}
$$

identities

$$
\begin{aligned}
& E(x, 0,0,0)=0 \\
& E(0, x, 0,0)=0 \\
& E(0,0, z, 0)=0 \\
& E(0,0,0, z)=0
\end{aligned}
$$

give that

$$
\begin{array}{r}
\chi_{1,2}(x)+\chi_{1,1}(x)=0 \\
\chi_{2,2}(x)+\chi_{2,1}(x)=0 \\
\zeta_{2,1}(z)+\zeta_{1,1}(z)=0 \\
\zeta_{2,2}(z)+\zeta_{1,2}(z)=0
\end{array} \quad(x \in X, z \in Y) .
$$

Moreover, equations

$$
\begin{aligned}
& E\left(x_{1}, 0, y_{1}, 0\right)=0 \\
& E\left(0, x_{2}, y_{1}, 0\right)=0 \\
& E\left(x_{1}, 0,0, y_{2}\right)=0 \\
& E\left(0, x_{2}, 0, y_{2}\right)=0
\end{aligned} \quad\left(x_{1}, x_{2} \in X, y_{1}, y_{2} \in Y\right)
$$

yield that

$$
f_{i, j}(x, z)=\chi_{i, j}(x)+\zeta_{i, j}(z) \quad(x \in X, z \in Y, i, j=1,2),
$$


where we used the previously proved identities, too.

3.1.2. The case $\alpha_{1}=\alpha_{2}=\beta_{1}=0$ and $\boldsymbol{\beta}_{2} \neq 0$. In such a situation (5) reduces to

$$
\begin{aligned}
& f\left(0, \beta_{2} y_{2}\right)=f_{1,1}\left(x_{1}, y_{1}\right)+f_{1,2}\left(x_{1}, y_{2}\right)+f_{2,1}\left(x_{2}, y_{1}\right)+f_{2,2}\left(x_{2}, y_{2}\right) \\
& \quad\left(x_{1}, x_{2} \in X, y_{1}, y_{2} \in Y\right) .
\end{aligned}
$$

Obviously, $\beta_{2}=1$ can be assumed, otherwise we consider the functions $\widetilde{f_{1,2}}, \widetilde{f_{2,2}}: X \times Y \rightarrow \mathbb{K}$ defined through

$$
\begin{aligned}
& \widetilde{f_{1,2}}(x, z)=f_{1,2}\left(x, \frac{z}{\beta_{2}}\right) \\
& \widetilde{f_{2,2}}(x, z)=f_{2,2}\left(x, \frac{z}{\beta_{2}}\right)
\end{aligned} \quad(x \in X, z \in Y) .
$$

Proposition 3. Let $X$ and $Y$ be linear spaces over the field $\mathbb{K}$ and $f, f_{1,1}, f_{1,2}$, $f_{2,1}, f_{2,2}: X \times Y \rightarrow \mathbb{K}$ be functions such that

$$
\begin{aligned}
& f\left(0, y_{2}\right)=f_{1,1}\left(x_{1}, y_{1}\right)+f_{1,2}\left(x_{1}, y_{2}\right)+f_{2,1}\left(x_{2}, y_{1}\right)+f_{2,2}\left(x_{2}, y_{2}\right) \\
& \quad\left(x_{1}, x_{2} \in X, y_{1}, y_{2} \in Y\right) .
\end{aligned}
$$

Then and only then for all $i, j=1,2$ there exist functions $\chi_{i, j}: X \rightarrow \mathbb{K}$ and $\zeta_{i, j}: Y \rightarrow \mathbb{K}$ such that

$$
f_{i, j}(x, z)=\chi_{i, j}(x)+\zeta_{i, j}(z) \quad(x \in X, z \in Y, i, j=1,2)
$$

as well as

$$
\begin{aligned}
\chi_{1,2}(x)+\chi_{1,1}(x) & =0 \\
\chi_{2,2}(x)+\chi_{2,1}(x) & =0 \\
\zeta_{2,1}(z)+\zeta_{1,1}(z) & =0 \\
\zeta_{2,2}(z)+\zeta_{1,2}(z) & =f(0, z)
\end{aligned} \quad(x \in X, z \in Y) .
$$

Proof. For $i, j \in\{1,2\}$ let us define the functions $\chi_{i, j}: X \rightarrow \mathbb{K}$ and $\zeta_{i, j}: Y \rightarrow$ $\mathbb{K}$ through

$$
\chi_{i, j}(x)=f_{i, j}(x, 0) \quad \text { and } \quad \zeta_{i, j}(z)=f_{i, j}(0, z) \quad(x \in X, z \in Y) .
$$

Furthermore, let

$$
\begin{aligned}
E & \left(x_{1}, x_{2}, y_{1}, y_{2}\right) \\
= & f\left(0, y_{2}\right)-f_{1,1}\left(x_{1}, y_{1}\right)-f_{1,2}\left(x_{1}, y_{2}\right)-f_{2,1}\left(x_{2}, y_{1}\right)-f_{2,2}\left(x_{2}, y_{2}\right) \\
& \left(x_{1}, x_{2} \in X, y_{1}, y_{2} \in Y\right)
\end{aligned}
$$

to obtain the following system of equations

$$
\begin{aligned}
& E\left(x_{1}, 0, y_{1}, 0\right)=0 \\
& E\left(x_{1}, 0,0, y_{2}\right)=0 \\
& E\left(0, x_{2}, y_{1}, 0\right)=0 \\
& E\left(0, x_{2}, 0, y_{2}\right)=0
\end{aligned} \quad\left(x_{1}, x_{2} \in X, y_{1}, y_{2} \in Y\right)
$$


or equivalently

$$
\begin{aligned}
& f_{1,1}\left(x_{1}, y_{1}\right)+f_{1,2}\left(x_{1}, 0\right)+f_{2,1}\left(0, y_{1}\right)=0 \\
& f_{1,2}\left(x_{1}, y_{2}\right)+f_{1,1}\left(x_{1}, 0\right)+f_{2,2}\left(0, y_{2}\right)=f\left(0, y_{2}\right) \\
& f_{2,1}\left(x_{2}, y_{1}\right)+f_{2,2}\left(x_{2}, 0\right)+f_{1,1}\left(0, y_{1}\right)=0 \\
& f_{2,2}\left(x_{2}, y_{2}\right)+f_{2,1}\left(x_{2}, 0\right)+f_{1,2}\left(0, y_{2}\right)=f\left(0, y_{2}\right)
\end{aligned}
$$

for all $x_{1}, x_{2} \in X, y_{1}, y_{2} \in Y$. Finally, the system of equations

$$
\begin{aligned}
& E\left(x_{1}, 0,0,0\right)=0 \\
& E\left(0, x_{2}, 0,0\right)=0 \\
& E\left(0,0, y_{1}, 0\right)=0 \\
& E\left(0,0,0, y_{2}\right)=0
\end{aligned} \quad\left(x_{1}, x_{2} \in X, y_{1}, y_{2} \in Y\right)
$$

yields that

$$
\begin{aligned}
& f_{1,2}\left(x_{1}, 0\right)+f_{1,1}\left(x_{1}, 0\right)=0 \\
& f_{2,2}\left(x_{2}, 0\right)+f_{2,1}\left(x_{2}, 0\right)=0 \\
& f_{2,1}\left(0, y_{1}\right)+f_{1,1}\left(0, y_{1}\right)=0 \\
& f_{2,2}\left(0, y_{2}\right)+f_{1,2}\left(0, y_{2}\right)=f\left(0, y_{2}\right)
\end{aligned} \quad\left(x_{1}, x_{2} \in X, y_{1}, y_{2} \in Y\right),
$$

which in view of the above definitions completes the proof.

3.1.3. The case $\boldsymbol{\alpha}_{1}, \boldsymbol{\alpha}_{2} \neq \mathbf{0}$ and $\boldsymbol{\beta}_{1}, \boldsymbol{\beta}_{2}=\mathbf{0}$. In such a situation (5) implies that

$$
\begin{gathered}
f\left(\alpha_{1} x_{1}+\alpha_{2} x_{2}, 0\right)=f_{1,1}\left(x_{1}, 0\right)+f_{1,2}\left(x_{1}, 0\right)+f_{2,1}\left(x_{2}, 0\right)+f_{2,2}\left(x_{2}, 0\right) \\
\left(x_{1}, x_{2} \in X\right)
\end{gathered}
$$

because the left hand side does not depend on $y_{1}$ and $y_{2}$.

Obviously, $\alpha_{1}, \alpha_{2}=1$ can be assumed, otherwise we consider the functions $\widetilde{f_{1,2}}, \widetilde{f_{2,2}}: X \times Y \rightarrow \mathbb{K}$ defined through

$$
\begin{aligned}
& \widetilde{f_{1,1}}(x, z)=f_{1,1}\left(\frac{x}{\alpha_{1}}, z\right) \\
& \widetilde{f_{1,2}}(x, z)=f_{1,2}\left(\frac{x}{\alpha_{1}}, z\right) \\
& \widetilde{f_{2,1}}(x, z)=f_{2,1}\left(\frac{x}{\alpha_{2}}, z\right) \\
& \widetilde{f_{2,2}}(x, z)=f_{2,2}\left(\frac{x}{\alpha_{2}}, z\right)
\end{aligned} \quad(x \in X, z \in Y) .
$$

Proposition 4. Let $X$ and $Y$ be linear spaces over the field $\mathbb{K}$ and $f, f_{1,1}, f_{1,2}$, $f_{2,1}, f_{2,2}: X \times Y \rightarrow \mathbb{K}$ be functions such that

$$
\begin{gathered}
f\left(x_{1}+x_{2}, 0\right)=f_{1,1}\left(x_{1}, 0\right)+f_{1,2}\left(x_{1}, 0\right)+f_{2,1}\left(x_{2}, 0\right)+f_{2,2}\left(x_{2}, 0\right) \\
\left(x_{1}, x_{2} \in X\right) .
\end{gathered}
$$

Then and only then there exists an additive function $\chi: X \rightarrow \mathbb{K}$ such that

$$
\begin{aligned}
f(x, 0) & =\chi(x) \\
f_{1,1}(x, 0)+f_{1,2}(x, 0) & =\chi(x) \\
f_{2,1}(x, 0)+f_{2,2}(x, 0) & =\chi(x)
\end{aligned} \quad(x \in X) .
$$


Proof. Consider the functions $\chi, \varphi, \psi: X \rightarrow \mathbb{K}$ defined through

$$
\begin{aligned}
& \chi(x)=f(x, 0) \\
& \varphi(x)=f_{1,1}(x, 0)+f_{1,2}(x, 0) \\
& \psi(x)=f_{2,1}(x, 0)+f_{2,2}(x, 0)
\end{aligned} \quad(x \in X)
$$

to get the following Pexider equation

$$
\chi\left(x_{1}+x_{2}\right)=\varphi\left(x_{1}\right)+\psi\left(x_{2}\right) \quad\left(x_{1}, x_{2} \in X\right) .
$$

Since all the functions $\chi, \varphi, \psi$ vanish at zero, we get that $\varphi \equiv \psi \equiv \chi$ and the function $\chi$ has to be additive.

To finish the discussion of Eq. (5) in this special case, apply Proposition 2 to the functions

$$
\tilde{f}_{i, j}(x, y)=f_{i, j}(x, y)-f_{i, j}(x, 0), \quad(x \in X, y \in Y)
$$

where $f_{i, j}(x, 0)$ were determined in Proposition 4.

3.1.4. The case $\boldsymbol{\alpha}_{1}, \boldsymbol{\beta}_{1} \neq \mathbf{0}$ and $\boldsymbol{\alpha}_{2}, \boldsymbol{\beta}_{2}=\mathbf{0}$. In such a situation (5) implies that

$$
f\left(\alpha_{1} x_{1}, \beta_{1} y_{1}\right)=f_{1,1}\left(x_{1}, y_{1}\right)+f_{1,2}\left(x_{1}, 0\right)+f_{2,1}\left(0, y_{1}\right) \quad\left(x_{1} \in X, y_{1} \in Y\right)
$$

because the left hand side does not depend on $x_{2}$ and $y_{2}$.

Obviously, due to similar reasons as previously, $\alpha_{1}, \beta_{1}=1$ can be assumed. The proof of the following proposition is a straightforward calculation, so we omit it.

Proposition 5. Let $X$ and $Y$ be linear spaces over the field $\mathbb{K}$ and $f, f_{1,1}, f_{1,2}$, $f_{2,1}: X \times Y \rightarrow \mathbb{K}$ be functions. The functional equation

$$
f\left(x_{1}, y_{1}\right)=f_{1,1}\left(x_{1}, y_{1}\right)+f_{1,2}\left(x_{1}, 0\right)+f_{2,1}\left(0, y_{1}\right) \quad\left(x_{1} \in X, y_{1} \in Y\right) .
$$

is fulfilled if and only if there exist functions $\chi: X \rightarrow \mathbb{K}$ and $\zeta: Y \rightarrow \mathbb{K}$ such that

$$
\begin{aligned}
f_{1,2}(x, 0) & =\chi(x) \\
f_{2,1}(0, z) & =\zeta(z) \\
f(x, z)-f_{1,1}(x, z) & =\chi(x)+\zeta(z)
\end{aligned} \quad(x \in X, z \in Y) .
$$

To finish the discussion of Eq. (5) in this special case, apply Proposition 2 to the functions

$$
\begin{aligned}
& \widetilde{f}_{1,1}(x, y)=0 \\
& \widetilde{f}_{1,2}(x, y)=f_{1,2}(x, y)-f_{1,2}(x, 0), \\
& \widetilde{f}_{2,1}(x, y)=f_{2,1}(x, y)-f_{2,1}(0, y), \quad(x \in X, y \in Y), \\
& \widetilde{f}_{2,2}(x, y)=f_{2,2}(x, y)
\end{aligned}
$$

where $f_{1,2}(x, 0)$ and $f_{2,1}(0, y)$ are given in Proposition 5 . 
3.1.5. The case $\boldsymbol{\alpha}_{1}, \boldsymbol{\alpha}_{2}, \boldsymbol{\beta}_{1} \neq \mathbf{0}$ and $\boldsymbol{\beta}_{\mathbf{2}}=\mathbf{0}$. In such a situation (5) implies that

$$
\begin{gathered}
f\left(\alpha_{1} x_{1}+\alpha_{2} x_{2}, \beta_{1} y_{1}\right)=f_{1,1}\left(x_{1}, y_{1}\right)+f_{1,2}\left(x_{1}, 0\right)+f_{2,1}\left(x_{2}, y_{1}\right)+f_{2,2}\left(x_{2}, 0\right) \\
\left(x_{1} \in X, y_{1} \in Y\right),
\end{gathered}
$$

because the left hand side does not depend on $y_{2}$.

Obviously, due to similar reasons as previously, $\alpha_{1}, \alpha_{2}, \beta_{1}=1$ can be assumed.

Proposition 6. Let $X$ and $Y$ be linear spaces over the field $\mathbb{K}$ and $f, f_{1,1}, f_{1,2}$, $f_{2,1}, f_{2,2}: X \times Y \rightarrow \mathbb{K}$ be functions. The functional equation

$$
\begin{aligned}
& f\left(x_{1}+x_{2}, y_{1}\right)=f_{1,1}\left(x_{1}, y_{1}\right)+f_{1,2}\left(x_{1}, 0\right)+f_{2,1}\left(x_{2}, y_{1}\right)+f_{2,2}\left(x_{2}, 0\right) \\
& \quad\left(x_{1}, x_{2} \in X, y_{1} \in Y\right)
\end{aligned}
$$

is fulfilled if and only if there exists a mapping $A: X \times Y \rightarrow \mathbb{K}$ additive in its first variable and there are functions $\chi, \chi_{1,1}, \chi_{2,1}: X \rightarrow \mathbb{K}$ and $\zeta, \zeta_{1,1}, \zeta_{2,1}: Y$ $\rightarrow \mathbb{K}$ vanishing at zero so that $\chi$ is additive and

$$
\begin{aligned}
f(x, z) & =A(x, z)+\chi(x)+\zeta(z) \\
f_{1,1}(x, z) & =A(x, z)+\chi_{1,1}(x)+\zeta_{1,1}(z) \\
f_{2,1}(x, z) & =A(x, z)+\chi_{2,1}(x)+\zeta_{2,1}(z)
\end{aligned} \quad(x \in X, z \in Y)
$$

and also

$$
\begin{aligned}
\chi(x) & =f(x, 0) \\
\chi_{1,1}(x) & =f_{1,1}(x, 0) \\
\chi_{2,1}(x) & =f_{2,1}(x, 0) \\
\zeta(z) & =\zeta_{1,1}(z)+\zeta_{2,1}(z) \quad(x \in X, z \in Y) \\
f_{1,2}(x, 0) & =\chi(x)-\chi_{1,1}(x) \\
f_{2,2}(x, 0) & =\chi(x)-\chi_{2,1}(x)
\end{aligned}
$$

hold.

Proof. With the substitution $y_{1}=0$ our equation yields that

$$
\begin{aligned}
& f\left(x_{1}+x_{2}, 0\right)=f_{1,1}\left(x_{1}, 0\right)+f_{1,2}\left(x_{1}, 0\right)+f_{2,1}\left(x_{2}, 0\right)+f_{2,2}\left(x_{2}, 0\right) \\
& \quad\left(x_{1}, x_{2} \in X,\right) .
\end{aligned}
$$

From this we immediately get that

$$
\tilde{f}\left(x_{1}+x_{2}, y_{1}\right)=\tilde{f}_{1,1}\left(x_{1}, y_{1}\right)+\tilde{f}_{2,1}\left(x_{2}, y_{1}\right) \quad\left(x_{1}, x_{2} \in X, y_{1} \in Y\right),
$$

where the functions $\widetilde{f}, \widetilde{f}_{1,1}, \widetilde{f}_{2,1}: X \times Y \rightarrow \mathbb{K}$ are defined by

$$
\begin{aligned}
\widetilde{f}(x, y) & =f(x, y)-f(x, 0) \\
\widetilde{f}_{1,1}(x, y) & =f_{1,1}(x, y)-f_{1,1}(x, 0) \\
\widetilde{f}_{2,1}(x, y) & =f_{2,1}(x, y)-f_{2,1}(x, 0)
\end{aligned} \quad(x \in X, y \in Y) .
$$


This means that the functions $\tilde{f}, \widetilde{f}_{1,1}, \widetilde{f}_{2,1}$ fulfill a Pexider equation on $X$ for any fixed $y \in Y$. Thus there exists a mapping $A: X \times Y \rightarrow \mathbb{K}$ additive in its first variable and functions $\zeta, \zeta_{1,1}, \zeta_{2,1}: Y \rightarrow \mathbb{K}$ such that

$$
\zeta(z)=\zeta_{1,1}(z)+\zeta_{2,1}(z) \quad(z \in Y)
$$

and

$$
\begin{aligned}
\widetilde{f}(x, z) & =A(x, z)+\zeta(z) \\
\widetilde{f}_{1,1}(x, z) & =A(x, z)+\zeta_{1,1}(z) \\
\widetilde{f}_{2,1}(x, z) & =A(x, z)+\zeta_{2,2}(z)
\end{aligned} \quad(x \in X, z \in Y) .
$$

In terms of the functions $f, f_{1,1}, f_{2,1}$ this means that

$$
\begin{aligned}
f(x, z) & =A(x, z)+\chi(x)+\zeta(z) \\
f_{1,1}(x, z) & =A(x, z)+\chi_{1,1}(x)+\zeta_{1,1}(z) \\
f_{2,1}(x, z) & =A(x, z)+\chi_{2,1}(x)+\zeta_{2,2}(z)
\end{aligned} \quad(x \in X, z \in Y),
$$

where

$$
\begin{aligned}
\chi(x) & =f(x, 0) \\
\chi_{1,1}(x) & =f_{1,1}(x, 0) \\
\chi_{2,1}(x) & =f_{2,1}(x, 0)
\end{aligned} \quad(x \in X) .
$$

Observe that $\chi$ is additive. Indeed, using the above, the forms of $f, f_{1,1}$ and $f_{2,2}$, our equation with $y_{1}=0$ and the fact that $A$ is additive in its first variable, we obtain that

$\chi\left(x_{1}+x_{2}\right)=\chi_{1,1}\left(x_{1}\right)+f_{1,2}\left(x_{1}, 0\right)+\chi_{2,1}\left(x_{2}\right)+f_{2,2}\left(x_{2}, 0\right) \quad\left(x_{1}, x_{2} \in X\right)$,

that is, $\chi$ fulfills a Pexider equation. Since $\chi(0)=0$, this means that $\chi$ has to be additive. Thus, using again the form of the functions $f, f_{1,1}, f_{2,1}$ and our equation with $x_{2}=0$, we get that

$$
f_{1,2}(x, 0)=\chi(x)-\chi_{1,1}(x) \quad(x \in X) .
$$

Similarly, our equation with $x_{1}=0$ implies that

$$
f_{2,2}(x, 0)=\chi(x)-\chi_{2,1}(x) \quad(x \in X) .
$$

To finish the discussion of Eq. (5) in this special case, apply Proposition 2 to the functions

$$
\begin{aligned}
& \widetilde{f}_{11}(x, y)=0, \\
& \widetilde{f}_{1,2}(x, y)=f_{1,2}(x, y)-f_{1,2}(x, 0), \quad(x \in X, y \in Y) \\
& \widetilde{f}_{2,1}(x, y)=0, \\
& \widetilde{f}_{2,2}(x, y)=f_{2,2}(x, y)-f_{2,2}(x, 0),
\end{aligned}
$$

where $f_{1,2}(x, 0)$ and $f_{2,2}(x, 0)$ are given in Proposition 6 . 


\subsection{The non-degenerate case}

After making the degenerate cases clear, we can now focus on the case $\alpha_{1}$, $\alpha_{2}, \beta_{1}, \beta_{2} \neq 0$ and provide the general solution of the functional equation

$$
\begin{aligned}
f & \left(\alpha_{1} x_{1}+\alpha_{2} x_{2}, \beta_{1} y_{1}+\beta_{2} y_{2}\right) \\
= & f_{1,1}\left(x_{1}, y_{1}\right)+f_{1,2}\left(x_{1}, y_{2}\right)+f_{2,1}\left(x_{2}, y_{1}\right)+f_{2,2}\left(x_{2}, y_{2}\right) \\
& \left(x_{1}, x_{2} \in X, y_{1}, y_{2} \in Y\right),
\end{aligned}
$$

where $f, f_{1,1}, f_{1,2}, f_{2,1}, f_{2,2}: X \times Y \rightarrow \mathbb{K}$ denote the unknown functions and $\alpha_{1}, \alpha_{2}, \beta_{1}, \beta_{2} \in \mathbb{K}$ are given constants.

Obviously, it is enough to consider the case $\alpha_{1}=\alpha_{2}=\beta_{1}=\beta_{2}=1$, that is, to consider the following functional equation

$$
\begin{aligned}
f & \left(x_{1}+x_{2}, y_{1}+y_{2}\right) \\
= & f_{1,1}\left(x_{1}, y_{1}\right)+f_{1,2}\left(x_{1}, y_{2}\right)+f_{2,1}\left(x_{2}, y_{1}\right)+f_{2,2}\left(x_{2}, y_{2}\right) \\
& \left(x_{1}, x_{2} \in X, y_{1}, y_{2} \in Y\right) .
\end{aligned}
$$

In this subsection we always assume that the characteristic of the field $\mathbb{K}$ is different from 2.

Proposition 7. Let $X$ and $Y$ be linear spaces over the field $\mathbb{K}$. Then functions $f, f_{1,1}, f_{1,2}, f_{2,1}, f_{2,2}: X \times Y \rightarrow \mathbb{K}$ satisfy the functional equation

$$
\begin{aligned}
f & \left(x_{1}+x_{2}, y_{1}+y_{2}\right) \\
= & f_{1,1}\left(x_{1}, y_{1}\right)+f_{1,2}\left(x_{1}, y_{2}\right)+f_{2,1}\left(x_{2}, y_{1}\right)+f_{2,2}\left(x_{2}, y_{2}\right) \\
& \left(x_{1}, x_{2} \in X, y_{1}, y_{2} \in Y\right) .
\end{aligned}
$$

if and only if

$$
\begin{aligned}
f(x, z) & =A(x, z)+\chi(x)+\zeta(z) \\
f_{i, j}(x, z) & =A(x, z)+\chi_{i, j}(x)+\zeta_{i, j}(z)
\end{aligned} \quad(x \in X, z \in Z),
$$

where the mapping $A: X \times Y \rightarrow \mathbb{K}$ is a bi-additive function and for $i, j \in\{1,2\}$ $\chi, \chi_{i, j}: X \rightarrow \mathbb{K}$ as well as $\zeta, \zeta_{i, j}: Y \rightarrow \mathbb{K}$ are functions such that $\chi$ and $\zeta$ are additive functions and $\chi_{i, j}$ and $\zeta_{i, j}$ vanish at the point $(0,0)$ and

$$
\begin{aligned}
& \chi(x)=\chi_{1,1}(x)+\chi_{1,2}(x)=\chi_{2,1}(x)+\chi_{2,2}(x) \\
& \zeta(z)=\zeta_{1,1}(z)+\zeta_{2,1}(z)=\zeta_{1,2}(z)+\zeta_{2,2}(z)
\end{aligned} \quad(x \in X, z \in Y)
$$

are also fulfilled.

Proof. Assume that the functions $f, f_{1,1}, f_{1,2}, f_{2,1}, f_{2,2}: X \times Y \rightarrow \mathbb{K}$ fulfill functional Eq. (7) for any $x_{1}, x_{2} \in X$ and $y_{1}, y_{2} \in Y$. With the substitution $y_{2}=0$ we obtain that

$$
\begin{aligned}
f & \left(x_{2}+x_{1}, y_{1}\right) \\
= & f_{2,1}\left(x_{2}, y_{1}\right)+f_{2,2}\left(x_{2}, 0\right)+f_{1,1}\left(x_{1}, y_{1}\right)+f_{1,2}\left(x_{1}, 0\right) \\
& \left(x_{1}, x_{2} \in X, y_{1} \in Y\right),
\end{aligned}
$$


which immediately implies that

$$
f\left(x_{2}+x_{1}, y_{1}\right)=\widetilde{f_{2,1}}\left(x_{2}, y_{1}\right)+\widetilde{f_{1,1}}\left(x_{1}, y_{1}\right) \quad\left(x_{1}, x_{2} \in X, y_{1} \in Y\right),
$$

where the functions $\widetilde{f_{1,1}}, \widetilde{f_{2,1}}$ are defined by

$$
\begin{aligned}
& \widetilde{f_{1,1}}(x, z)=f_{1,1}(x, z)+f_{1,2}(x, 0) \\
& \widetilde{f_{2,1}}(x, z)=f_{2,1}(x, z)+f_{2,2}(x, 0)
\end{aligned} \quad(x \in X, z \in Y) .
$$

This means that there exists a function $A^{(1)}: X \times Y \rightarrow \mathbb{K}$ which is additive in its first variable and a function $\zeta: Y \rightarrow \mathbb{K}$ vanishing at zero such that

$$
f(x, z)=A^{(1)}(x, z)+\zeta(z) \quad(x \in X, z \in Y) .
$$

Substituting this form into Eq. (7), with $x_{2}=0$ and a similar argument we receive that

$$
A^{(1)}(x, z)=A(x, z)+\chi(x) \quad(x \in X, z \in Y),
$$

where $A: X \times Y \rightarrow \mathbb{K}$ is a bi-additive mapping and $\chi: X \rightarrow \mathbb{K}$ is a function that vanishes at zero.

All in all this means that

$$
f(x, z)=A(x, z)+\chi(x)+\zeta(z) \quad(x \in X, z \in Y) .
$$

Additionally, Eq. (7), first with $y_{1}=y_{2}=0$ yields that $\chi$ has to be additive and secondly, with $x_{1}=x_{2}=0$ we receive that the function $\zeta$ also has to be additive.

Define functions $F_{1,1}, F_{1,2}, F_{2,1}, F_{2,2}$ on $X \times Y$ through

$$
\begin{aligned}
& F_{1,1}(x, z)=f_{1,1}(x, z)-A(x, z)-\frac{\chi(x)}{2}-\frac{\zeta(z)}{2} \\
& F_{1,2}(x, z)=f_{1,2}(x, z)-A(x, z)-\frac{\chi(x)}{2}-\frac{\zeta(z)}{2} \\
& F_{2,1}(x, z)=f_{2,1}(x, z)-A(x, z)-\frac{\chi(x)}{2}-\frac{\zeta(z)}{2} \\
& F_{2,2}(x, z)=f_{2,2}(x, z)-A(x, z)-\frac{\chi(x)}{2}-\frac{\zeta(z)}{2}
\end{aligned}
$$

to deduce that they fulfill functional Eq. (6). Due to Proposition 2, for all $i, j=1,2$ there exist functions $\tilde{\chi}_{i, j}: X \rightarrow \mathbb{K}$ and $\widetilde{\zeta}_{i, j}: Y \rightarrow \mathbb{K}$ vanishing at zero such that

$$
F_{i, j}(x, z)=\widetilde{\chi}_{i, j}(x)+\widetilde{\zeta}_{i, j}(z) \quad(x \in X, z \in Y, i, j=1,2),
$$

that is, for the functions $f_{i, j}$ we have

$$
f_{i, j}(x, z)=A(x, z)+\chi_{i, j}(x)+\zeta_{i, j}(z) \quad(i, j \in\{1,2\}, x \in X, z \in Y) .
$$


Finally, using the equations in Proposition 2 for the functions $\widetilde{\chi}_{i, j}$ and $\widetilde{\zeta}_{i, j}$, the identities

$$
\begin{aligned}
& f(x, 0)=\chi(x)=f_{1,1}(x, 0)+f_{1,2}(x, 0)=\chi_{1,1}(x)+\chi_{1,2}(x) \\
& f(x, 0)=\chi(x)=f_{2,1}(x, 0)+f_{2,2}(x, 0)=\chi_{2,1}(x)+\chi_{2,2}(x) \\
& f(0, z)=\zeta(z)=f_{1,1}(0, z)+f_{2,1}(0, z)=\zeta_{1,1}(z)+\zeta_{2,1}(z) \\
& f(0, z)=\zeta(z)=f_{1,2}(0, z)+f_{2,2}(0, z)=\zeta_{1,2}(z)+\zeta_{2,2}(z)
\end{aligned}
$$

fulfilled by all $x \in X, z \in Y$ complete the proof.

\subsection{Related equations}

3.3.1. The functional equation of bi-additivity. As a trivial consequence of the results of the previous section we get the following.

Corollary 1. Let $X$ and $Y$ be linear spaces over the field $\mathbb{K}$ with char $(\mathbb{K}) \neq 2$. A mapping $f: X \times Y \rightarrow \mathbb{K}$ fulfills the functional equation of bi-additivity, that is,

$$
\begin{aligned}
f\left(x_{1}+x_{2}, y_{1}+y_{2}\right) & \\
= & f\left(x_{1}, y_{1}\right)+f\left(x_{1}, y_{2}\right)+f\left(x_{2}, y_{1}\right)+f\left(x_{2}, y_{2}\right) \\
& \left(x_{1}, x_{2} \in X, y_{1}, y_{2} \in Y\right)
\end{aligned}
$$

if and only if $f$ is bi-additive.

3.3.2. The rectangle equation. Let $X$ and $Y$ be linear spaces over the field $\mathbb{K}$ and let $f: X \times Y \rightarrow \mathbb{K}$ be a function.

Then the functional equation

$$
\begin{aligned}
& f(x+u, y+v)+f(x+u, y-v) \\
& \quad+f(x-u, y+v)+f(x-u, y-v)=4 f(x, y) \\
& \quad(x, y \in X, u, v \in Y)
\end{aligned}
$$

or equivalently (provided that $\operatorname{char}(\mathbb{K}) \neq 2$ )

$$
\begin{aligned}
4 f & \left(\frac{x_{1}+x_{2}}{2}, \frac{y_{1}+y_{2}}{2}\right) \\
= & f\left(x_{1}, y_{1}\right)+f\left(x_{1}, y_{2}\right)+f\left(x_{2}, y_{1}\right)+f\left(x_{2}, y_{2}\right) \\
& \left(x_{1}, x_{2} \in X, y_{1}, y_{2} \in Y\right) .
\end{aligned}
$$

is called the rectangle equation.

Indeed, both of the above equations express the following: the value of $f$ at the center of any rectangle, with parallel sides to the coordinate axes, equals the mean of the values of $f$ at the vertices.

This equation as well as its generalization were investigated (among others) in $[2,5,14]$.

With the aid of the results of the previous section, we obtain the following straightaway. 
Proposition 8. Let $X$ and $Y$ be linear spaces over the field $\mathbb{K}$ with char $(\mathbb{K}) \neq 2$ and $f: X \times Y \rightarrow \mathbb{K}$ be a function. The function $f$ fulfills the rectangle equation, i.e.,

$$
\begin{aligned}
4 f & \left(\frac{x_{1}+x_{2}}{2}, \frac{y_{1}+y_{2}}{2}\right) \\
= & f\left(x_{1}, y_{1}\right)+f\left(x_{1}, y_{2}\right)+f\left(x_{2}, y_{1}\right)+f\left(x_{2}, y_{2}\right) \\
& \left(x_{1}, x_{2} \in X, y_{1}, y_{2} \in Y\right),
\end{aligned}
$$

if and only if there exists a bi-additive mapping $A: X \times Y \rightarrow \mathbb{K}$ and additive functions $\chi: X \rightarrow \mathbb{K}$ and $\zeta: Y \rightarrow \mathbb{K}$ such that

$$
f(x, z)=A(x, z)+\chi(x)+\zeta(z) \quad(x \in X, z \in Y) .
$$

Remark. In case char $(\mathbb{K})=2$, the rectangle equation reduces to the equation

$$
\begin{array}{r}
f(x+u, y+v)+f(x+u, y-v) \\
+f(x-u, y+v)+f(x-u, y-v)=0 \quad(x, y \in X, u, v \in Y),
\end{array}
$$

or equivalently

$f\left(x_{1}, y_{1}\right)+f\left(x_{1}, y_{2}\right)+f\left(x_{2}, y_{1}\right)+f\left(x_{2}, y_{2}\right)=0 \quad\left(x_{1}, x_{2} \in X, y_{1}, y_{2} \in Y\right)$.

Thus, Proposition 2 yields that there exist functions $\chi: X \rightarrow \mathbb{K}$ and $\zeta: Y \rightarrow \mathbb{K}$ such that

$$
f(x, y)=\chi(x)+\zeta(y) \quad(x \in X, y \in Y) .
$$

The Cauchy equation on $X \times Y$.

Proposition 9. Let $X$ and $Y$ be linear spaces over the field $\mathbb{K}$ and $f, g, h: X \times$ $Y \rightarrow \mathbb{K}$ be functions. Then the functional equation

$$
f\left(x_{1}+x_{2}, y_{1}+y_{2}\right)=g\left(x_{1}, y_{1}\right)+h\left(x_{2}, y_{2}\right) \quad\left(x_{1}, x_{2} \in X, y_{1}, y_{2} \in Y\right)
$$

holds if and only if there exist additive functions $\chi: X \rightarrow \mathbb{K}, \zeta: Y \rightarrow \mathbb{K}$ such that

$$
\begin{aligned}
& f(x, y)=\chi(x)+\zeta(z) \\
& g(x, y)=\chi(x)+\zeta(z) \\
& h(x, y)=\chi(x)+\zeta(z)
\end{aligned} \quad(x \in X, z \in Y) .
$$

\section{On the reduction of equations with $n>2$ to the two-variable case}

In this section we intend to investigate the following problem. Let $X$ and $Y$ be linear spaces over the field $\mathbb{K}$, let further $\alpha_{i}, \beta_{i} \in \mathbb{K}, i=1, \ldots, n$ be arbitrarily 
fixed constants. Assume further that for functions $f, f_{i, j}: X \times Y \rightarrow \mathbb{K}, i, j=$ $1, \ldots, n$, the functional equation

$$
f\left(\sum_{i=1}^{n} \alpha_{i} x_{i}, \sum_{i=1}^{n} \beta_{i} y_{i}\right)=\sum_{i, j=1}^{n} f_{i, j}\left(x_{i}, y_{j}\right) \quad\left(x_{i} \in X, y_{i} \in Y, i=1, \ldots, n\right)
$$

is fulfilled.

We will show that in case $n>2$, the results of the previous section can be applied. Indeed, let $\lambda, \kappa, \mu, \nu \in\{1, \ldots, n\}$ such that $\lambda \neq \kappa$ and $\mu \neq \nu$, but otherwise arbitrary. In this case Eq. (9) with the substitutions

$$
x_{i}=0 \text { if } i \neq \lambda, \kappa \quad \text { and } \quad y_{j}=0 \text { if } j \neq \mu, \nu
$$

yields that

$$
\begin{aligned}
f( & \left.\alpha_{\lambda} x_{\lambda}+\alpha_{\kappa} x_{\kappa}, \beta_{\mu} y_{\mu}+\beta_{\nu} y_{\nu}\right) \\
= & f_{\lambda, \mu}\left(x_{\lambda}, y_{\mu}\right)+f_{\lambda, \nu}\left(x_{\lambda}, y_{\nu}\right)+f_{\kappa, \mu}\left(x_{\kappa}, y_{\mu}\right)+f_{\kappa, \nu}\left(x_{\kappa}, y_{\nu}\right) \\
& +\sum_{j \neq \mu, \nu} f_{\lambda, j}\left(x_{\lambda}, 0\right)+\sum_{j \neq \mu, \nu} f_{\kappa, j}\left(x_{\kappa}, 0\right) \\
& +\sum_{i \neq \lambda, \kappa} f_{i, \mu}\left(0, y_{\mu}\right)+\sum_{i \neq \lambda, \kappa} f_{i, \nu}\left(0, y_{\nu}\right)
\end{aligned}
$$

for any $x_{\lambda}, x_{\kappa} \in X$ and $y_{\mu}, y_{\nu} \in Y$. Consider the functions $\widetilde{f_{\lambda, \mu}}, \widetilde{f_{\kappa, \nu}}: X \times Y \rightarrow$ $\mathbb{K}$ defined by

$$
\widetilde{f_{\lambda, \mu}}(x, z)=f_{\lambda, \mu}(x, z)+\sum_{j \neq \mu, \nu} f_{\lambda, j}(x, 0)+\sum_{i \neq \lambda, \kappa} f_{i, \mu}(0, z) \quad(x \in X, z \in Y)
$$

and

$$
\widetilde{f_{\kappa, \nu}}(x, z)=f_{\kappa, \nu}(x, z)+\sum_{j \neq \mu, \nu} f_{\kappa, j}(x, 0)+\sum_{i \neq \lambda, \kappa} f_{i, \nu}(0, z) \quad(x \in X, z \in Y)
$$

to receive that

$$
\begin{aligned}
f & \left(\alpha_{\lambda} x_{\lambda}+\alpha_{\kappa} x_{\kappa}, \beta_{\mu} y_{\mu}+\beta_{\nu} y_{\nu}\right) \\
& =\widetilde{f_{\lambda, \mu}}\left(x_{\lambda}, y_{\mu}\right)+f_{\lambda, \nu}\left(x_{\lambda}, y_{\nu}\right)+f_{\kappa, \mu}\left(x_{\kappa}, y_{\mu}\right)+\widetilde{f_{\kappa, \nu}}\left(x_{\kappa}, y_{\nu}\right)
\end{aligned}
$$

is satisfied for any $x_{\lambda}, x_{\kappa} \in X$ and $y_{\mu}, y_{\nu} \in Y$. This equation can however be handled with the aid of the results of Sect. 3 . 


\section{The case of a single unknown function in the equation: existence of non-trivial solutions}

Let $X$ and $Y$ be linear spaces over the same field $\mathbb{K}$ and consider the following functional equation

$$
\begin{aligned}
f( & \left(\alpha_{1} x_{1}+\alpha_{2} x_{2}, \beta_{1} y_{1}+\beta_{2} y_{2}\right) \\
= & \gamma_{1,1} f\left(x_{1}, y_{1}\right)+\gamma_{1,2} f\left(x_{1}, y_{2}\right)+\gamma_{2,1} f\left(x_{2}, y_{1}\right)+\gamma_{2,2} f\left(x_{2}, y_{2}\right) \\
& \left(x_{1}, x_{2} \in X, y_{1}, y_{2} \in Y\right),
\end{aligned}
$$

where $f: X \times Y \rightarrow \mathbb{K}$ denotes the unknown function and $\alpha_{1}, \alpha_{2}, \beta_{1}, \beta_{2} \in \mathbb{K}$ and $\gamma_{1,1}, \gamma_{1,2}, \gamma_{2,1}, \gamma_{2,2} \in \mathbb{K}$ are given constants.

Recall that due to the linearity of the above equation we may (and we also do) suppose that

$$
f(0,0)=0
$$

holds. Otherwise the function

$$
\widetilde{f}(x, y)=f(x, y)-f(0,0) \quad(x \in X, y \in Y)
$$

can be considered. This function clearly vanishes at the point $(0,0)$ and it fulfills the same functional equation, too.

Furthermore, the linearity of the investigated equation implies that the identically zero function is always a solution. In this section we would like to study under what conditions Eq. (11) admits a non-identically zero solution. Clearly, in every case the results of the previous sections can be applied with the choice

$$
f_{i, j}(x, y)=\gamma_{i, j} f(x, y) \quad(x \in X, y \in Y) .
$$

This means that the assumption that the function $f$ is not identically zero will imply algebraic conditions for the involved parameters $\alpha_{1}, \alpha_{2}, \beta_{1}, \beta_{2} \in \mathbb{K}$ and $\gamma_{1,1}, \gamma_{1,2}, \gamma_{2,1}, \gamma_{2,2} \in \mathbb{K}$

Similarly as before, first we consider the so-called degenerate cases.

\subsection{Degenerate cases}

5.1.1. The case $\boldsymbol{\alpha}_{\mathbf{1}}=\boldsymbol{\alpha}_{\mathbf{2}}=\boldsymbol{\beta}_{\mathbf{1}}=\boldsymbol{\beta}_{\mathbf{2}}=\mathbf{0}$. In case $\alpha_{1}=\alpha_{2}=\beta_{1}=\beta_{2}=0$ Eq. (11) reduces to

$$
\begin{aligned}
& \gamma_{1,1} f\left(x_{1}, y_{1}\right)+\gamma_{1,2} f\left(x_{1}, y_{2}\right)+\gamma_{2,1} f\left(x_{2}, y_{1}\right)+\gamma_{2,2} f\left(x_{2}, y_{2}\right)=0 \\
& \quad\left(x_{1}, x_{2} \in X, y_{1}, y_{2} \in Y\right),
\end{aligned}
$$

where $\gamma_{i, j} \in \mathbb{K}$ for any $i, j \in\{1,2\}$. 
Proposition 10. Let $X$ and $Y$ be linear spaces over the field $\mathbb{K}, \gamma_{i, j} \in \mathbb{K}$ be given constants such that not all of them are zero and $f: X \times Y \rightarrow \mathbb{K}$ be a function such that

$$
\begin{aligned}
& \gamma_{1,1} f\left(x_{1}, y_{1}\right)+\gamma_{1,2} f\left(x_{1}, y_{2}\right)+\gamma_{2,1} f\left(x_{2}, y_{1}\right)+\gamma_{2,2} f\left(x_{2}, y_{2}\right)=0 \\
& \quad\left(x_{1}, x_{2} \in X, y_{1}, y_{2} \in Y\right) .
\end{aligned}
$$

Then and only then there exist functions $\chi: X \rightarrow \mathbb{K}$ and $\zeta: Y \rightarrow \mathbb{K}$ vanishing at zero such that

$$
f(x, z)=\chi(x)+\zeta(z) \quad(x \in X, z \in Y) .
$$

Furthermore

(i) either the following system of linear equations

$$
\begin{aligned}
& \gamma_{1,1}+\gamma_{1,2}=0 \\
& \gamma_{2,1}+\gamma_{2,2}=0
\end{aligned}
$$

is fulfilled or the function $\chi$ is identically zero.

(ii) either the following system of linear equations

$$
\begin{aligned}
& \gamma_{1,1}+\gamma_{2,1}=0 \\
& \gamma_{1,2}+\gamma_{2,2}=0
\end{aligned}
$$

is fulfilled or the function $\zeta$ is identically zero.

Proof. In view of Proposition 2 we get that there exist functions $\chi: X \rightarrow \mathbb{K}$ and $\zeta: Y \rightarrow \mathbb{K}$ vanishing at zero such that

$$
f(x, z)=\chi(x)+\zeta(z) \quad(x \in X, z \in Y) .
$$

Using this representation of the function $f$, Eq. (12) yields that

$$
\begin{aligned}
& \gamma_{1,1}\left(\chi\left(x_{1}\right)+\zeta\left(y_{1}\right)\right)+\gamma_{1,2}\left(\chi\left(x_{1}\right)+\zeta\left(y_{2}\right)\right) \\
& +\gamma_{2,1}\left(\chi\left(\left(x_{2}\right)+\zeta\left(y_{1}\right)\right)+\gamma_{2,2}\left(\chi\left(x_{2}\right)+\zeta\left(y_{2}\right)\right)=0\right. \\
& \quad\left(x_{1}, x_{2} \in X, y_{1}, y_{2} \in Y\right),
\end{aligned}
$$

or equivalently

$$
\begin{aligned}
& \chi\left(x_{1}\right)\left(\gamma_{1,1}+\gamma_{1,2}\right)+\chi\left(x_{2}\right)\left(\gamma_{2,1}+\gamma_{2,2}\right) \\
& \quad+\zeta\left(y_{1}\right)\left(\gamma_{1,1}+\gamma_{2,1}\right)+\zeta\left(y_{2}\right)\left(\gamma_{1,2}+\gamma_{2,2}\right)=0 \\
& \quad\left(x_{1}, x_{2} \in X, y_{1}, y_{2} \in Y\right) .
\end{aligned}
$$

Since we have independent variables, we get that

$$
\begin{aligned}
& \gamma_{1,1}+\gamma_{1,2}=0 \\
& \gamma_{2,1}+\gamma_{2,2}=0
\end{aligned}
$$

is fulfilled or the function $\chi$ is identically zero. Similarly,

$$
\begin{aligned}
& \gamma_{1,1}+\gamma_{2,1}=0 \\
& \gamma_{1,2}+\gamma_{2,2}=0
\end{aligned}
$$

holds or the function $\zeta$ is identically zero. 
5.1.2. The case $\alpha_{1}=\alpha_{2}=\beta_{1}=0$ and $\beta_{2} \neq 0$. In such a situation (11) reduces to

$$
\begin{gathered}
f\left(0, \beta_{2} y_{2}\right)=\gamma_{1,1} f\left(x_{1}, y_{1}\right)+\gamma_{1,2} f\left(x_{1}, y_{2}\right)+\gamma_{2,1} f\left(x_{2}, y_{1}\right)+\gamma_{2,2} f\left(x_{2}, y_{2}\right) \\
\left(x_{1}, x_{2} \in X, y_{1}, y_{2} \in Y\right) .
\end{gathered}
$$

In view of Proposition 3, the proof of the following proposition is straightforward and similar to that of Proposition 10. The basic step is to consider $f$ as the sum of single variable functions (Proposition 3) and substitute such a special form of $f$ into the functional equation.

Proposition 11. Let $X$ and $Y$ be linear spaces over the field $\mathbb{K}, \beta_{2}, \gamma_{i, j} \in \mathbb{K}$ be given constants such that not all of them are zero, and $f: X \times Y \rightarrow \mathbb{K}$ be a function such that

$$
\begin{gathered}
f\left(0, \beta_{2} y_{2}\right)=\gamma_{1,1} f\left(x_{1}, y_{1}\right)+\gamma_{1,2} f\left(x_{1}, y_{2}\right)+\gamma_{2,1} f\left(x_{2}, y_{1}\right)+\gamma_{2,2} f\left(x_{2}, y_{2}\right) \\
\left(x_{1}, x_{2} \in X, y_{1}, y_{2} \in Y\right) .
\end{gathered}
$$

Then and only then there exist functions $\chi: X \rightarrow \mathbb{K}$ and $\zeta: Y \rightarrow \mathbb{K}$ vanishing at zero such that

$$
f(x, z)=\chi(x)+\zeta(z) \quad(x \in X, z \in Y) .
$$

Furthermore

(i) either the following system of linear equations

$$
\begin{aligned}
& \gamma_{1,1}+\gamma_{1,2}=0 \\
& \gamma_{2,1}+\gamma_{2,2}=0
\end{aligned}
$$

is fulfilled or the function $\chi$ is identically zero.

(ii) either the following system of equations

$$
\begin{aligned}
\gamma_{1,1}+\gamma_{2,1} & =0 \\
\zeta\left(\beta_{2} z\right) & =\left(\gamma_{1,2}+\gamma_{2,2}\right) \zeta(z)
\end{aligned}
$$

is fulfilled or the function $\zeta$ is identically zero.

5.1.3. The case $\alpha_{1}, \alpha_{2} \neq 0$ and $\boldsymbol{\beta}_{1}, \boldsymbol{\beta}_{2}=0$. In such a situation (11) reduces to

$$
\begin{aligned}
f( & \left.\alpha_{1} x_{1}+\alpha_{2} x_{2}, 0\right) \\
= & \gamma_{1,1} f\left(x_{1}, y_{1}\right)+\gamma_{1,2} f\left(x_{1}, y_{2}\right)+\gamma_{2,1} f\left(x_{2}, y_{1}\right)+\gamma_{2,2} f\left(x_{2}, y_{2}\right) \\
& \left(x_{1}, x_{2} \in X, y_{1}, y_{2} \in Y\right) .
\end{aligned}
$$

As before, taking $f$ as the sum of single variable functions (Proposition 4), substitute it into the functional equation. 
Proposition 12. Let $X$ and $Y$ be linear spaces over the field $\mathbb{K}, \alpha_{1}, \alpha_{2}, \gamma_{i, j} \in \mathbb{K}$ be given constants such that not all of them are zero and $f: X \times Y \rightarrow \mathbb{K}$ be a function such that

$$
\begin{aligned}
f( & \left.\alpha_{1} x_{1}+\alpha_{2} x_{2}, 0\right) \\
= & \gamma_{1,1} f\left(x_{1}, y_{1}\right)+\gamma_{1,2} f\left(x_{1}, y_{2}\right)+\gamma_{2,1} f\left(x_{2}, y_{1}\right)+\gamma_{2,2} f\left(x_{2}, y_{2}\right) \\
& \left(x_{1}, x_{2} \in X, y_{1}, y_{2} \in Y\right) .
\end{aligned}
$$

Then and only then there exists an additive function $a: X \rightarrow \mathbb{K}$ and a function $\zeta: Y \rightarrow \mathbb{K}$ vanishing at zero such that

$$
f(x, y)=a(x)+\zeta(y) \quad(x \in X, y \in Y) .
$$

Furthermore the above additive function a has to fulfill

$$
a\left(\alpha_{1} x_{1}+\alpha_{2} x_{2}\right)=\left(\gamma_{1,1}+\gamma_{1,2}\right) a\left(x_{1}\right)+\left(\gamma_{2,1}+\gamma_{2,2}\right) a\left(x_{2}\right)
$$

for arbitrary $x_{1}, x_{2} \in X$ and for the mapping $\zeta$ alternative (ii) of Proposition 10 is fulfilled.

5.1.4. The case $\boldsymbol{\alpha}_{1}, \boldsymbol{\beta}_{1} \neq \mathbf{0}$ and $\boldsymbol{\alpha}_{2}, \boldsymbol{\beta}_{2}=\mathbf{0}$. In such a situation (11) reduces to

$$
\begin{aligned}
f( & \left.\alpha_{1} x_{1}, \beta_{1} y_{1}\right) \\
= & \gamma_{1,1} f\left(x_{1}, y_{1}\right)+\gamma_{1,2} f\left(x_{1}, y_{2}\right)+\gamma_{2,1} f\left(x_{2}, y_{1}\right)+\gamma_{2,2} f\left(x_{2}, y_{2}\right) \\
& \left(x_{1}, x_{2} \in X, y_{1}, y_{2} \in Y\right) .
\end{aligned}
$$

To prove the following result, consider $f$ as the sum of single variable functions (Proposition 5) and substitute it into the functional equation.

Proposition 13. Let $X$ and $Y$ be linear spaces over the field $\mathbb{K}, \alpha_{1}, \alpha_{2}, \gamma_{i, j} \in \mathbb{K}$ be given constants and $f: X \times Y \rightarrow \mathbb{K}$ be a function such that

$$
\begin{aligned}
& f\left(\alpha_{1} x_{1}, \beta_{1} y_{1}\right) \\
& =\gamma_{1,1} f\left(x_{1}, y_{1}\right)+\gamma_{1,2} f\left(x_{1}, y_{2}\right)+\gamma_{2,1} f\left(x_{2}, y_{1}\right)+\gamma_{2,2} f\left(x_{2}, y_{2}\right) \\
& \quad\left(x_{1}, x_{2} \in X, y_{1}, y_{2} \in Y\right) .
\end{aligned}
$$

Then and only then

(A) $\gamma_{1,2}, \gamma_{2,1}, \gamma_{2,2}=0$ then $f: X \times Y \rightarrow \mathbb{K}$ is an arbitrary function fulfilling

$$
f\left(\alpha_{1} x, \beta_{1} y\right)=\gamma_{1,1} f(x, y) \quad(x \in X, y \in Y),
$$

(B) or there exist functions $\chi: X \rightarrow \mathbb{K}$ and $\zeta: Y \rightarrow \mathbb{K}$ vanishing at zero such that

$$
f(x, y)=\chi(x)+\zeta(y) \quad(x \in X, z \in Y) .
$$

Furthermore the mappings $\chi$ and $\zeta$ also fulfill

$$
\begin{aligned}
\chi\left(\alpha_{1} x\right) & =\left(\gamma_{1,1}+\gamma_{1,2}\right) \chi(x) \\
\zeta\left(\beta_{1} z\right) & =\left(\gamma_{1,1}+\gamma_{2,1}\right) \zeta(z)
\end{aligned} \quad(x \in X, z \in Y)
$$

and 
(i) either

$$
\gamma_{2,1}+\gamma_{2,2}=0
$$

or $\chi$ is identically zero;

(ii) either

$$
\gamma_{1,2}+\gamma_{2,2}=0
$$

or $\zeta$ is identically zero.

5.1.5. The case $\boldsymbol{\alpha}_{1}, \boldsymbol{\alpha}_{2}, \boldsymbol{\beta}_{1} \neq \mathbf{0}$ and $\boldsymbol{\beta}_{2}=\mathbf{0}$. In such a situation (11) reduces to

$$
\begin{aligned}
f( & \left.\alpha_{1} x_{1}+\alpha_{2} x_{2}, \beta_{1} y_{1}\right) \\
= & \gamma_{1,1} f\left(x_{1}, y_{1}\right)+\gamma_{1,2} f\left(x_{1}, y_{2}\right)+\gamma_{2,1} f\left(x_{2}, y_{1}\right)+\gamma_{2,2} f\left(x_{2}, y_{2}\right) \\
& \left(x_{1}, x_{2} \in X, y_{1}, y_{2} \in Y\right) .
\end{aligned}
$$

Proposition 14. Let $X$ and $Y$ be linear spaces over the field $\mathbb{K}, \alpha_{1}, \alpha_{2}, \beta_{1}, \gamma_{i, j}$ $\in \mathbb{K}, i, j=1,2$ be given constants and $f: X \times Y \rightarrow \mathbb{K}$ be a function such that

$$
\begin{aligned}
& f\left(\alpha_{1} x_{1}+\alpha_{2} x_{2}, \beta_{1} y_{1}\right) \\
& =\gamma_{1,1} f\left(x_{1}, y_{1}\right)+\gamma_{1,2} f\left(x_{1}, y_{2}\right)+\gamma_{2,1} f\left(x_{2}, y_{1}\right)+\gamma_{2,2} f\left(x_{2}, y_{2}\right) \\
& \quad\left(x_{1}, x_{2} \in X, y_{1}, y_{2} \in Y\right) .
\end{aligned}
$$

Then and only then there exists a mapping $A: X \times Y \rightarrow \mathbb{K}$ additive in its first variable, further there are functions $\chi: X \rightarrow \mathbb{K}$ and $\zeta: Y \rightarrow \mathbb{K}$ vanishing at zero such that $\chi$ is additive and

$$
f(x, y)=A(x, y)+\chi(x)+\zeta(y) \quad(x \in X, y \in Y) .
$$

Furthermore, we have that

$$
\zeta\left(\beta_{1} y\right)=\left(\gamma_{1,1}+\gamma_{2,1}\right) \zeta(y) \quad(y \in Y)
$$

and also

$$
\left(\gamma_{1,2}+\gamma_{2,2}\right) \zeta(y)=0 \quad(y \in Y),
$$

yielding that $\gamma_{1,2}+\gamma_{2,2}=0$ or $\zeta$ is identically zero. Additionally, the alternatives below also hold

(A) either $\gamma_{1,2}$ and $\gamma_{2,2}$ are zero, that is, Eq. (16) has the form

$$
\begin{aligned}
& f\left(\alpha_{1} x_{1}+\alpha_{2} x_{2}, \beta_{1} y_{1}\right) \\
& \quad=\gamma_{1,1} f\left(x_{1}, y_{1}\right)+\gamma_{2,1} f\left(x_{2}, y_{1}\right) \quad\left(x_{1}, x_{2} \in X, y_{1} \in Y\right)
\end{aligned}
$$

and the identities

$$
\begin{array}{ll}
A\left(\alpha_{1} x, \beta_{1} y\right)+\chi\left(\alpha_{1} x\right)=\gamma_{1,1} A(x, y)+\gamma_{1,1} \chi(x) & (x \in X, y \in Y) \\
\text { and } & \\
A\left(\alpha_{2} x, \beta_{1} y\right)+\chi\left(\alpha_{2} x\right)=\gamma_{2,1} A(x, y)+\gamma_{2,1} \chi(x) & (x \in X, y \in Y) \\
\text { have to hold; } &
\end{array}
$$


(B) or $\gamma_{1,2}$ and $\gamma_{2,2}$ do not vanish simultaneously and then the mapping $A$ has a rather special form, namely there exists an additive function $a: X \rightarrow \mathbb{K}$ such that

$$
A(x, y)=a(x) \quad(x \in X)
$$

and therefore

$$
f(x, y)=a(x)+\chi(x)+\zeta(y) \quad(x \in X, y \in Y)
$$

where the identities

$$
a\left(\alpha_{1} x\right)+\chi\left(\alpha_{1} x\right)=\gamma_{1,1} a(x)+\gamma_{1,1} \chi(x) \quad(x \in X, y \in Y)
$$

and

$$
a\left(\alpha_{2} x\right)+\chi\left(\alpha_{2} x\right)=\gamma_{2,1} a(x)+\gamma_{2,1} \chi(x) \quad(x \in X, y \in Y)
$$

have to hold.

Proof. Using Proposition 6 we immediately get that there exists a mapping $A: X \times Y \rightarrow \mathbb{K}$ and there are functions $\chi: X \rightarrow \mathbb{K}$ and $\zeta: Y \rightarrow \mathbb{K}$ vanishing at zero such that $\chi$ is additive and

$$
f(x, y)=A(x, y)+\chi(x)+\zeta(y) \quad(x \in X, y \in Y) .
$$

Using that $A$ is additive in its first variable and Proposition 7, from Eq. (16) we derive that

$$
\begin{aligned}
& A\left(\alpha_{1} x_{1}, \beta_{1} y_{1}\right)+A\left(\alpha_{2} x_{2}, \beta_{1} y_{1}\right)+\chi\left(\alpha_{1} x_{1}+\alpha_{2} x_{2}\right)+\zeta\left(\beta_{1} y_{1}\right) \\
& =\gamma_{1,1} A\left(x_{1}, y_{1}\right)+\gamma_{1,1} \chi\left(x_{1}\right)+\gamma_{1,1} \zeta\left(y_{1}\right) \\
& \quad+\gamma_{1,2} A\left(x_{1}, y_{2}\right)+\gamma_{1,2} \chi\left(x_{1}\right)+\gamma_{1,2} \zeta\left(y_{2}\right) \\
& \quad+\gamma_{2,1} A\left(x_{2}, y_{1}\right)+\gamma_{2,1} \chi\left(x_{2}\right)+\gamma_{2,1} \zeta\left(y_{1}\right) \\
& \quad+\gamma_{2,2} A\left(x_{2}, y_{2}\right)+\gamma_{2,2} \chi\left(x_{2}\right)+\gamma_{2,2} \zeta\left(y_{2}\right) \\
& \quad\left(x_{1}, x_{2} \in X, y_{1}, y_{2} \in Y\right) .
\end{aligned}
$$

Observe that this equation with $x_{1}=x_{2}=y_{1}=0$ implies that

$$
\left(\gamma_{1,2}+\gamma_{2,2}\right) \zeta\left(y_{2}\right)=0 \quad\left(y_{2} \in Y\right)
$$

so $\gamma_{1,2}+\gamma_{2,2}=0$ or the function $\zeta$ is identically zero. Similarly, Eq. (17) yields with $x_{1}=x_{2}=y_{2}=0$ that

$$
\zeta\left(\beta_{1} y_{1}\right)=\left(\gamma_{1,1}+\gamma_{2,1}\right) \zeta\left(y_{1}\right) \quad\left(y_{1} \in Y\right) .
$$

Here while proving the last two identities we used that $\chi(0)=\zeta(0)=0$ (cf. the proof of Proposition 6 ) and the fact that $A(0, y)=0$ for all $y \in Y$ since $A$ is additive in its first variable. Put $x_{2}=y_{1}=0$ into (17) to obtain that

$$
\begin{aligned}
& A\left(\alpha_{1} x_{1}, 0\right)+\chi\left(\alpha_{1} x_{1}\right) \\
& =\gamma_{1,1} A\left(x_{1}, 0\right)+\gamma_{1,1} \chi\left(x_{1}\right)+\gamma_{1,2} A\left(x_{1}, y_{2}\right)+\gamma_{1,2} \chi\left(x_{1}\right) \\
& \quad\left(x_{1}, x_{2} \in X, y_{1}, y_{2} \in Y\right)
\end{aligned}
$$


or in other words,

$$
\begin{aligned}
- & \gamma_{1,2} A\left(x_{1}, y_{2}\right) \\
= & \gamma_{1,1} A\left(x_{1}, 0\right)+\gamma_{1,1} \chi\left(x_{1}\right)+\gamma_{1,2} \chi\left(x_{1}\right)-A\left(\alpha_{1} x_{1}, 0\right)-\chi\left(\alpha_{1} x_{1}\right) \\
& \left(x_{1} \in X, y_{2} \in Y\right) .
\end{aligned}
$$

Similarly Eq. (17) with $x_{1}=y_{1}=0$ yields that

$$
\begin{aligned}
& A\left(\alpha_{2} x_{2}, 0\right)+\chi\left(\alpha_{2} x_{2}\right) \\
& =\gamma_{2,1} A\left(x_{2}, 0\right)+\gamma_{2,1} \chi\left(x_{2}\right)+\gamma_{2,2} A\left(x_{2}, y_{2}\right)+\gamma_{2,2} \chi\left(x_{2}\right) \\
& \quad\left(x_{2} \in X, y_{2} \in Y\right)
\end{aligned}
$$

or equivalently

$$
\begin{aligned}
- & \gamma_{2,2} A\left(x_{2}, y_{2}\right) \\
= & \gamma_{2,1} A\left(x_{2}, 0\right)+\gamma_{2,1} \chi\left(x_{2}\right)+\gamma_{2,2} \chi\left(x_{2}\right)-A\left(\alpha_{2} x_{2}, 0\right)-\chi\left(\alpha_{2} x_{2}\right) \\
& \left(x_{2} \in X, y_{2} \in Y\right) .
\end{aligned}
$$

From these latter two identities the following alternatives can be deduced

(A) either $\gamma_{1,2}$ and $\gamma_{2,2}$ are zero and Eq. (16) has the form

$$
f\left(\alpha_{1} x_{1}+\alpha_{2} x_{2}, \beta_{1} y_{1}\right)=\gamma_{1,1} f\left(x_{1}, y_{1}\right)+\gamma_{2,1} f\left(x_{2}, y_{1}\right) \quad\left(x_{1}, x_{2} \in X, y_{1} \in Y\right)
$$

and the identities

$$
A\left(\alpha_{1} x, \beta_{1} y\right)+\chi\left(\alpha_{1} x\right)=\gamma_{1,1} A(x, y)+\gamma_{1,1} \chi(x) \quad(x \in X, y \in Y)
$$

as well as

$$
A\left(\alpha_{2} x, \beta_{1} y\right)+\chi\left(\alpha_{2} x\right)=\gamma_{2,1} A(x, y)+\gamma_{2,1} \chi(x) \quad(x \in X, y \in Y)
$$

follow immediately from (17) with $x_{2}=y_{2}=0$ and $x_{1}=y_{2}=0$, respectively.

(B) or the two-variable mapping $A$ can be represented as

$$
A(x, y)=a(x) \quad(x \in X, y \in Y) ;
$$

$A$ being additive in its first variable. This is possible if and only if $a: X \rightarrow$ $\mathbb{K}$ is additive. This means that

$$
f(x, y)=a(x)+\chi(x)+\zeta(y) \quad(x \in X, y \in Y) .
$$

Using this representation and Eq. (14) first with $x_{2}=y_{2}=0$ and after that with $x_{1}=y_{2}=0$ we get the identities

$$
a\left(\alpha_{1} x\right)+\chi\left(\alpha_{1} x\right)=\gamma_{1,1} a(x)+\gamma_{1,1} \chi(x) \quad(x \in X, y \in Y)
$$

and

$$
a\left(\alpha_{2} x\right)+\chi\left(\alpha_{2} x\right)=\gamma_{2,1} a(x)+\gamma_{2,1} \chi(x) \quad(x \in X, y \in Y) .
$$




\subsection{The non-degenerate case}

In view of the above results, now we can focus on the case $\alpha_{1}, \alpha_{2}, \beta_{1}, \beta_{2} \neq 0$ and investigate the existence of nontrivial solutions of functional equation

$$
\begin{aligned}
& f\left(\alpha_{1} x_{1}+\alpha_{2} x_{2}, \beta_{1} y_{1}+\beta_{2} y_{2}\right) \\
& =\gamma_{1,1} f\left(x_{1}, y_{1}\right)+\gamma_{1,2} f\left(x_{1}, y_{2}\right)+\gamma_{2,1} f\left(x_{2}, y_{1}\right)+\gamma_{2,2} f\left(x_{2}, y_{2}\right) \\
& \quad\left(x_{1}, x_{2} \in X, y_{1}, y_{2} \in Y\right),
\end{aligned}
$$

where for $i, j \in\{1,2\}$, the constants $\gamma_{i, j} \in \mathbb{K}$ are given.

Here we will make use of the results of Sect. 3.2, therefore (as in Sect. 3.2) we always assume that the characteristic of the field $\mathbb{K}$ is different from 2 . As a direct application of Proposition 7 we derive the following.

Proposition 15. Let $X$ and $Y$ be linear spaces over the field $\mathbb{K}, \alpha_{i}, \beta_{j}, \gamma_{i, j} \in \mathbb{K}$, $i, j=1,2$ be given constants and $f: X \times Y \rightarrow \mathbb{K}$ be a function such that

$$
\begin{aligned}
f( & \left(\alpha_{1} x_{1}+\alpha_{2} x_{2}, \beta_{1} y_{1}+\beta_{2} y_{2}\right) \\
= & \gamma_{1,1} f\left(x_{1}, y_{1}\right)+\gamma_{1,2} f\left(x_{1}, y_{2}\right)+\gamma_{2,1} f\left(x_{2}, y_{1}\right)+\gamma_{2,2} f\left(x_{2}, y_{2}\right) \\
& \left(x_{1}, x_{2} \in X, y_{1}, y_{2} \in Y\right) .
\end{aligned}
$$

Then and only then, there exist a bi-additive function $A: X \times Y \rightarrow \mathbb{K}$ and additive functions $\chi: X \rightarrow \mathbb{K}$ and $\zeta: Y \rightarrow \mathbb{K}$ such that

$$
f(x, z)=A(x, z)+\chi(x)+\zeta(z) \quad(x \in X, z \in Y) .
$$

Furthermore the following identities also have to be fulfilled

$$
\begin{aligned}
\chi\left(\alpha_{1} x\right) & =\left(\gamma_{1,1}+\gamma_{1,2}\right) \chi(x) \\
\chi\left(\alpha_{2} x\right) & =\left(\gamma_{2,1}+\gamma_{2,2}\right) \chi(x) \\
\zeta\left(\beta_{1} z\right) & =\left(\gamma_{1,1}+\gamma_{2,1}\right) \zeta(z) \\
\zeta\left(\beta_{2} z\right) & =\left(\gamma_{1,2}+\gamma_{2,2}\right) \zeta(z) \\
A\left(\alpha_{i} x, \beta_{j} z\right) & =\gamma_{i, j} A(x, y)
\end{aligned} \quad(x \in X, z \in Y, i, j=1,2) .
$$

Remark. While investigating whether Eq. (11) admits a non-trivial solution or not we always got three types of conditions. One of them is a purely algebraic condition, namely we have to check if the parameters $\gamma_{i, j}$ fulfill a system of homogeneous, linear equations.

The second type is about the existence of a non-trivial semi-homogeneous additive function. More precisely, this condition is always of the following form: let $X$ be a linear space over the field $\mathbb{K}$ and let $a: X \rightarrow \mathbb{K}$ be an additive function such that

$$
a(\alpha x)=\beta a(x) \quad(x \in X)
$$

with certain fixed scalars $\alpha, \beta \in \mathbb{K}$. For which values of $\alpha$ and $\beta$ will the function $a$ be non-trivial (that is, non-identically zero)? This question was first investigated in Daróczy [6] if $X=\mathbb{K}=\mathbb{R}$. These results were later generalized and extended in the papers $[7-10,14,19,20]$. To the best of our knowledge, 
this problem has not been investigated for fields with nonzero characteristic. To this we provide a solution in Sect. 6.2.

Our third condition is similar to the second one, namely it concerns the nontriviality of a semi-homogeneous bi-additive function. The attached existence problem will also be discussed in the last section.

\section{A necessary and sufficient condition for the existence of non-zero, bi-additive semi-homogeneous mappings}

According to the characteristic property

$$
A\left(\alpha_{i} x, \beta_{j} y\right)=\gamma_{i, j} A(x, y) \quad(x \in X, y \in Y, i, j=1,2)
$$

of the bi-additive term in the solution (see Proposition 15), it is natural to investigate the problem of existence of such a non-zero mapping over the field $\mathbb{K}$.

Definition 4. Let $X$ and $Y$ be linear spaces over the field $\mathbb{K}$. An additive function $a: X \rightarrow \mathbb{K}$ is called semi-homogeneous if there exist elements $\alpha, \beta \in \mathbb{K}$ such that

$$
a(\alpha x)=\beta a(x) \quad(x \in X) .
$$

Similarly, a bi-additive function $A: X \times Y \rightarrow \mathbb{K}$ is called semi-homogeneous if there exist elements $\alpha, \beta$ and $\gamma$ in the field $\mathbb{K}$ such that

$$
A(\alpha x, \beta y)=\gamma A(x, y) \quad(x \in X, y \in Y) \text {. }
$$

\subsection{The case of fields with characteristic zero}

In this subsection we restrict ourselves to the case of fields with zero characteristic. According to this, let $\mathbb{K}$ be a field of characteristic zero. Then it is an extension of the field $\mathbb{Q}$ of the rationals and we can consider the subfields $\mathbb{Q}(\alpha)$ and $\mathbb{Q}(\beta)$ in $\mathbb{K}$.

We will also use the following.

Proposition 16. Let $\mathbb{K}$ be a field of characteristic zero. Then $\mathbb{K}$ is embeddable into $\mathbb{C}$ if and only if the transcendence degree of the field extension $\mathbb{K} / \mathbb{Q}$ is less than $\mathfrak{c}$.

Remark. From the previous proposition we immediately get that if $\mathbb{K}$ is finitely generated over $\mathbb{Q}$, that is if $\mathbb{K}=\mathbb{Q}\left(\alpha_{1}, \ldots, \alpha_{n}\right)$, then $\mathbb{K}$ is embeddable into $\mathbb{C}$.

Theorem 3. Let $\mathbb{K}$ be a field of characteristic zero and suppose that $X$ and $Y$ are linear spaces over $\mathbb{K}$. There exists a non-zero bi-additive mapping $A: X \times$ $Y \rightarrow \mathbb{K}$ satisfying (19) if and only if $\gamma$ can be written as the product of algebraically conjugated elements to $\alpha$ and $\beta$ over $\mathbb{Q}$, respectively. 
Proof. Suppose that $\gamma$ can be written as the product of algebraically conjugated elements to $\alpha$ and $\beta$ over $\mathbb{Q}$, respectively. This means that $\gamma=$ $\delta_{1}(\alpha) \delta_{2}(\beta)$, where $\delta_{1}: \mathbb{Q}(\alpha) \rightarrow \mathbb{K}$ and $\delta_{2}: \mathbb{Q}(\beta) \rightarrow \mathbb{K}$ are injective homomorphisms. Taking $X$ and $Y$ as linear spaces over $\mathbb{Q}(\alpha)$ and $\mathbb{Q}(\beta)$, respectively, we can write that

$$
x=\sum_{i \in I} \frac{p_{i}(\alpha)}{q_{i}(\alpha)} x_{i} \quad \text { and } \quad y=\sum_{j \in J} \frac{r_{j}(\beta)}{s_{j}(\beta)} y_{j},
$$

where

(i) $I$ and $J$ are finite index sets such that $|I|=k$ and $|J|=l$,

(ii) $p_{i}, q_{i} \in \mathbb{Q}[x]$ for any $i \in I$,

(iii) $r_{j}, s_{j} \in \mathbb{Q}[x]$ for any $j \in J$,

(iv) $x_{1}, \ldots, x_{k}$ belong to a basis of $X$ as a linear space over $\mathbb{Q}(\alpha)$,

(v) $y_{1}, \ldots, y_{l}$ belong to a basis of $Y$ as a linear space over $\mathbb{Q}(\beta)$.

The mapping $A$ is defined by the formula of the semi-linear extension

$$
A(x, y)=\sum_{i=1}^{k} \sum_{j=1}^{l} \frac{p_{i}\left(\delta_{1}(\alpha)\right)}{q_{i}\left(\delta_{1}(\alpha)\right)} \frac{r_{j}\left(\delta_{2}(\beta)\right)}{s_{j}\left(\delta_{2}(\beta)\right)} A\left(x_{i}, y_{j}\right)
$$

and the values $A\left(x_{i}, y_{j}\right)$ are not all zero.

Conversely, suppose that there exists a non-zero bi-additive mapping $A: X \times$ $Y \rightarrow \mathbb{K}$ satisfying (19). Let us fix elements $x \in X$ and $y \in Y$ such that $A(x, y) \neq 0$. Taking the field $\mathbb{L}=\mathbb{Q}(\alpha, \beta, A(x, y), \gamma)$ we can define the biadditive mapping $B: \mathbb{L} \times \mathbb{L} \rightarrow \mathbb{C}$ as

$$
B(u, v)=A(u x, v y) \quad(u, v \in \mathbb{L}) .
$$

It can be easily seen that

$$
B(\alpha u, \beta v)=\gamma B(u, v)
$$

is fulfilled for arbitrary $u, v \in \mathbb{L}$.

Let $\mathbb{L}^{*}$ denote the multiplicative subgroup of $\mathbb{L}$ and let $G=\mathbb{L}^{*} \times \mathbb{L}^{*}$ be the group equipped with pointwise multiplication. Then, for any $\left(u_{*}, v_{*}\right) \in G$, the translate mapping, that is,

$$
\left(\tau_{\left(u^{*}, v^{*}\right)} B\right)(u, v)=B\left(u u^{*}, v v^{*}\right)
$$

also satisfies (20). Let $V$ be the set of restrictions of bi-additive mappings of the form $B: \mathbb{L} \times \mathbb{L} \rightarrow \mathbb{C}$ satisfying (20). Then the set $V$ is closed with respect to uniform convergence on finite sets and the field $\mathbb{L}$ is countable. Therefore $V$ is a closed, translation invariant linear space, in other words, it is a variety over a field of finite transcendence degree. From this we infer that spectral analysis holds in $V$, i.e., there exists an exponential element in this variety, see Laczkovich-Székelyhidi [12]. An exponential element in this variety is a bi-additive mapping $M: \mathbb{L} \times \mathbb{L} \rightarrow \mathbb{C}$ satisfying (20) so that

$$
M\left(u u_{*}, v v_{*}\right)=M(u, v) M\left(u_{*}, v_{*}\right) .
$$


Using the notations $\delta_{1}(u)=M(u, 1)$ and $\delta_{2}(v)=M(1, v)$, it follows that $M(u, v)=\delta_{1}(u) \delta_{2}(v)$, where $\delta_{1}$ and $\delta_{2}$ are injective (field-) homomorphisms of L. Using property $(20)$

$$
\gamma M(u, v)=M(\alpha u, \beta v)=\delta_{1}(\alpha) \delta_{2}(\beta) M(u, v) .
$$

Therefore $\gamma=\delta_{1}(\alpha) \delta_{2}(\beta)$ as it was stated.

\subsection{The case of finite fields of non-zero characteristic}

If $\mathbb{K}$ is a field of characteristic different from zero then it is a field of prime characteristic. First of all we collect those results from the theory of finite fields, that we intend to use subsequently. Here we rely on the monograph Lidl-Niederreiter [13]. For any field $\mathbb{K}$, there is a minimal subfield, namely the prime field of $\mathbb{K}$, which is the smallest subfield containing 1 . It is isomorphic either to $\mathbb{Q}$ (if the characteristic is zero), or to a finite field of prime order $\mathbb{Z}_{p}$ (in case $\operatorname{char}(\mathbb{K})=p$ ). Moreover, if $p$ is a prime and $n \in \mathbb{N}$ is arbitrary, then up to isomorphism there exists exactly one finite field of order $q=p^{n}$. This field is nothing but the splitting field of the polynomial $x^{q}-x$ over $\mathbb{Z}_{p}$. This field is denoted by $\mathrm{GF}(q)$.

Let now $a: X \rightarrow \mathbb{K}$ be a semi-homogeneous additive function, that is, assume that for the additive function $a$ we have

$$
a(\alpha x)=\beta a(x) \quad(x \in X) .
$$

As we have seen in the proof of Theorem 3, the problem of existence of semi-homogeneous mappings defined on the linear space $X$ can be reduced to the problem of existence of semi-homogeneous mappings defined on $\mathbb{K}$. It can be easily seen that additivity automatically implies homogeneity with respect to multiplication by elements of the prime field. By the argument of [18], it also follows that there exists an automorphism between the extensions of the prime field with $\alpha$ and $\beta$, respectively, such that it maps $\alpha$ into $\beta$. Conversely, such an automorphism allows us to use the technique of semi-linear extension to construct semi-homogeneous additive mappings. This criteria for the existence of semi-homogeneous additive mappings does not depend on the characteristic of the fields but it is worth investigating the problem of subfields in $\mathbb{K}$ in some special cases as follows.

Let $\varphi: \mathbb{K} \rightarrow \mathbb{K}$ be an automorphism of $\mathbb{K}$ with $\varphi(\beta)=\alpha$. Then

$$
(\varphi \circ a)(\alpha x)=\varphi(\beta) \cdot(\varphi \circ a)(x)=\alpha \cdot(\varphi \circ a)(x) \quad(x \in X) .
$$

This means that

(i) we have to guarantee the existence of an automorphism $\varphi: \operatorname{GF}\left(p^{n}\right) \rightarrow$ $\mathrm{GF}\left(p^{n}\right)$ for which

$$
\varphi(\beta)=\alpha
$$


is satisfied;

(ii) we have to determine the homogeneity field (see Definition 6) of the additive mapping $\varphi \circ a: X \rightarrow \mathbb{K}$.

Suppose that $\mathbb{K} \simeq \operatorname{GF}\left(p^{n}\right)$ for some prime $p$ and $n \in \mathbb{N}$.

To answer the above questions, for (i) we have to know the automorphism group of $\operatorname{GF}\left(p^{n}\right)$, while for (ii) we have to describe the subfields of $\operatorname{GF}\left(p^{n}\right)$.

Definition 5. Let $p$ be a prime and $n \in \mathbb{N}$. By an automorphism $\varphi$ of $\operatorname{GF}\left(p^{n}\right)$ over $\operatorname{GF}(p)$ we mean an automorphism of $\operatorname{GF}\left(p^{n}\right)$ that fixes the elements of $\operatorname{GF}(p)$. More precisely, we require $\varphi$ to be a one-to-one mapping from $\operatorname{GF}\left(p^{n}\right)$ onto itself with

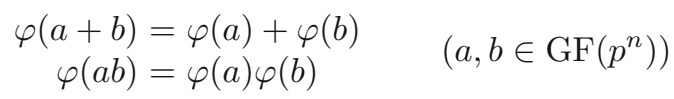

and

$$
\varphi(a)=a \quad(a \in \mathrm{GF}(p))
$$

Theorem 4. Let $p$ be a prime and $n \in \mathbb{N}$. The distinct automorphisms of $\operatorname{GF}\left(p^{n}\right)$ over $G F(p)$ are exactly the mappings $\varphi_{0}, \varphi_{1}, \ldots, \varphi_{n-1}$ defined by

$$
\varphi_{j}(a)=a^{p^{j}} \quad\left(a \in \mathrm{GF}\left(p^{n}\right), j=0,1, \ldots, n-1\right) .
$$

Remark. In other words, the above theorem says that the automorphism group of $\operatorname{GF}\left(p^{n}\right)$ over $\operatorname{GF}(p)$ is a cyclic group of order $n$ generated by $\varphi_{1}$.

Let $X$ be a linear space over the (not necessarily finite) field $\mathbb{K}$ and $a: X \rightarrow$ $\mathbb{K}$ be an additive function. Then clearly, for any $k \in \mathbb{Z}$ we have

$$
a(k x)=k a(x) \quad(x \in X) .
$$

Nevertheless, it may happen that $a$ satisfies the same identity for all $x \in X$ and also for some $\alpha \in \mathbb{K} \backslash \mathbb{Z}$, therefore we introduce the following.

Definition 6. Let $X$ be a linear space over the (not necessarily finite) field $\mathbb{K}$ and $a: X \rightarrow \mathbb{K}$ be an additive function and

$$
\mathbb{H}_{a}=\{\alpha \in \mathbb{K} \mid a(\alpha x)=\alpha a(x) \text { for all } x \in X\} .
$$

This set is called the homogeneity field of the additive function a. Observe that this term is well-motivated, since we have the following.

Although the following two statements are known in case $\mathbb{K}=\mathbb{R}$ (see Kuczma [11]), for the sake of completeness we present a short argument for them.

Proposition 17. Let $X$ be a linear space over the field $\mathbb{K}$ and $a: X \rightarrow \mathbb{K}$ be an additive function. Then $\mathbb{H}_{a} \subset \mathbb{K}$ is a field. 
Proof. Let $\alpha, \beta \in \mathbb{H}_{a}$, then

$$
a((\alpha-\beta) x)=a(\alpha x)-a(\beta x)=\alpha a(x)-\beta a(x)=(\alpha-\beta) a(x) \quad(x \in X),
$$

yielding that $\alpha-\beta \in \mathbb{H}_{a}$. Similarly, if $\beta \neq 0$, then

$$
\alpha a(x)=a(\alpha x)=a\left(\beta \frac{\alpha}{\beta} x\right)=\beta a\left(\frac{\alpha}{\beta} x\right) \quad(x \in X),
$$

from which $\frac{\alpha}{\beta} \in \mathbb{H}_{a}$ follows.

In some sense, the converse is also true, namely we have the proposition below. The proof is based on the existence of Hamel bases of linear spaces. Therefore, in any case it is needed, the Axiom of Choice is supposed to hold.

Proposition 18. Let $X$ be a linear space over the field $\mathbb{K}$, let further $\mathbb{L} \subset \mathbb{K}$ be a subfield of $\mathbb{K}$. Then there exists an additive function $a: X \rightarrow \mathbb{K}$ such that $\mathbb{H}_{a}=\mathbb{L}$.

Proof. Let $B$ be the Hamel basis of the linear space $(X, \mathbb{L},+, \cdot)$, which (according to Corollary 4.2.1. of Kuczma [11]) does exist. Fix $c \in \mathbb{K} \backslash\{0\}$ and define the function $f: B \rightarrow \mathbb{K}$ by

$$
f(x)=c \quad(x \in B) .
$$

By Theorem 4.3.1 of Kuczma [11], there exists a homomorphism $a$ from $(X, \mathbb{L},+, \cdot)$ to $(\mathbb{K}, \mathbb{L},+, \cdot)$ such that we additionally have that $\left.a\right|_{B}=f$. Clearly, $a$ is an additive function and

$$
a(\alpha x)=\alpha a(x) \quad(x \in X, \alpha \in \mathbb{L}) .
$$

Thus $\mathbb{L} \subset \mathbb{H}_{a}$.

For the converse statement, let $x \in X$ be arbitrary, then $x=\sum_{i=1}^{n} \lambda_{i} b_{i}$, where $\lambda_{i} \in \mathbb{L}$ and $b_{i} \in B$ for all $i=1, \ldots, n$. Furthermore,

$$
a(x)=a\left(\sum_{i=1}^{n} \lambda_{i} b_{i}\right)=\sum_{i=1}^{n} \lambda_{i} a\left(b_{i}\right)=\sum_{i=1}^{n} \lambda_{i} f\left(b_{i}\right)=c \cdot \sum_{i=1}^{n} \lambda_{i} \in c \cdot \mathbb{L},
$$

or equivalently, $a(X) \subset c \cdot \mathbb{L}$.

Let now $\alpha \in \mathbb{H}_{a}$ and $b_{0} \in B$ be arbitrary, then

$$
a\left(\alpha b_{0}\right)=\alpha a\left(b_{0}\right)=\alpha f\left(b_{0}\right)=\alpha c
$$

On the other hand, since $\alpha b_{0} \in X$, the inclusion $a(X) \subset c \cdot \mathbb{L}$ implies that there exists $\lambda \in \mathbb{L}$ such that $a\left(\alpha b_{0}\right)=\lambda c$. Since $c$ was to be chosen nonzero, this means that $\alpha=\lambda \in \mathbb{L}$. Therefore $\mathbb{H}_{a} \subset \mathbb{L}$.

Theorem 5. Let $p$ be a prime and $n \in \mathbb{N}$. Then for all $d \mid n$, the field $\operatorname{GF}\left(p^{n}\right)$ admits exactly one subfield isomorphic to $\mathrm{GL}\left(p^{d}\right)$ and $\mathrm{GL}\left(p^{n}\right)$ has no other type of subfields. Furthermore, this subfield is the set of zeros of the polynomial $x^{p^{d}}-x$ in $\operatorname{GF}\left(p^{n}\right)$. 
Finally, we provide necessary and sufficient conditions for the existence of non-zero, bi-additive semi-homogeneous mappings.

The relations among the elements $\alpha, \beta$ and $\gamma$ such that the semi-homogeneity Eq. (19) is satisfied for some non-zero bi-additive mapping $A: X \times Y \rightarrow \mathbb{K}$ are more implicit as we will see in what follows.

Lemma 1. Let $X$ and $Y$ be linear spaces over the field $\mathbb{K}$ and let $\alpha, \beta, \gamma \in \mathbb{K}$ be given non-zero elements. There exists a not identically zero bi-additive mapping $A: X \times Y \rightarrow \mathbb{K}$ satisfying the semi-homogeneity Eq. (19) if and only if there exists a not identically zero bi-additive mapping $B: \mathbb{K} \times \mathbb{K} \rightarrow \mathbb{K}$ satisfying equation

$$
B(\alpha u, \beta v)=\gamma B(u, v) \quad(\gamma \neq 0) .
$$

Proof. Suppose that $A: X \times Y \rightarrow \mathbb{K}$ satisfies the semi-homogeneity Eq. (19) and $A(x, y) \neq 0$ for a certain element $(x, y) \in X \times Y$. The bi-additive mapping $B: \mathbb{K} \times \mathbb{K} \rightarrow \mathbb{K}$ defined by

$$
B(u, v)=A(u x, v y) \quad(u, v \in \mathbb{K})
$$

obviously satisfies Eq. (21). Conversely, suppose that $B: \mathbb{K} \times \mathbb{K} \rightarrow \mathbb{K}$ satisfies Eq. (21). Let $\left\{x_{\mu}\right\}_{\mu \in \Gamma_{X}}$ and $\left\{y_{\nu}\right\}_{\nu \in \Gamma_{Y}}$ be Hamel bases in $X$ and $Y$, respectively. Taking the projections

$$
\pi_{X}^{1}: X \times Y \rightarrow \mathbb{K} \text { and } \pi_{Y}^{1}: X \times Y \rightarrow \mathbb{K}
$$

onto the first coordinate of the elements with respect to the given bases it follows that the mapping $A: X \times Y \rightarrow \mathbb{K}$ defined by

$$
A(x, y)=B\left(\pi_{X}^{1}(x), \pi_{Y}^{1}(y)\right) \quad(x \in X, y \in Y)
$$

fulfills (19).

Remark. Note that there is no need for any additional condition for the cardinality of the field $\mathbb{K}$ to prove Lemma 1.

From now on the results are strongly based on the cardinality condition for $\mathbb{K}$ being finite. Let $\mathbb{K}=\operatorname{GF}(q)$, where $q=p^{n}$ for some prime number $p \in \mathbb{N}$ and consider a (finite) basis $b_{0}, \ldots, b_{n-1}$ of $\mathbb{K}$ over its prime field $\mathbb{Z}_{p}$. It is clear that

(H) bi-additivity implies $\mathbb{Z}_{p}$-homogeneity for any bi-additive mapping $B$ : $\mathbb{K} \times \mathbb{K} \rightarrow \mathbb{K}$.

Since the translation $\tau_{i}: \mathbb{K} \rightarrow \mathbb{K}$ with respect to multiplication by the $i^{\text {th }}$ element of the given basis $(i=0, \ldots, n-1)$, that is,

$$
\tau_{i}(x)=b_{i} \cdot x \quad(x \in \mathbb{K})
$$


is a linear transformation, we can consider its matrix representation $M^{i}$ given by

$$
\tau_{i}\left(b_{k}\right)=\sum_{j=0}^{n-1} m_{j k}^{(i)} b_{j}
$$

where for any possible indices we have $m_{j k}^{(i)} \in \mathbb{Z}_{p}$. According to property $(\mathrm{H})$, a simple calculation shows that Eq. (21) is equivalent to

$$
\gamma B\left(b_{k}, b_{l}\right)=\sum_{i, j=0}^{n-1} \alpha_{i} \beta_{j} \sum_{r, s=0}^{n-1} m_{r k}^{(i)} m_{s l}^{(j)} B\left(b_{r}, b_{s}\right)
$$

where $k, l=0, \ldots n-1, \alpha=\sum_{i=0}^{n-1} \alpha_{i} b_{i}$ and $\beta=\sum_{j=0}^{n-1} \beta_{j} b_{j}$ with $\alpha_{i}, \beta_{j} \in \mathbb{Z}_{p}$.

Let $\mathscr{M}_{n}(\mathbb{K})$ be the linear space of matrices of order $n$ over the field $\mathbb{K}$ and consider the linear mapping

$$
P_{\alpha, \beta}: \mathscr{M}_{n}(\mathbb{K}) \ni X \mapsto Y=P_{\alpha, \beta}(X),
$$

where

$$
y_{k l}=\sum_{i, j=0}^{n-1} \alpha_{i} \beta_{j} \sum_{r, s=0}^{n-1} m_{r k}^{(i)} m_{s l}^{(j)} x_{r s}
$$

In a more compact form

$$
P_{\alpha, \beta}(X)=\sum_{i, j=0}^{n-1} \alpha_{i} \beta_{j}\left(M^{(i)}\right)^{T} X M^{(j)} .
$$

Equation (21) is obviously satisfied if and only if $0 \neq \gamma \in \mathbb{K}$ is an eigenvalue of $P_{\alpha, \beta}$. The corresponding (non-zero) eigenvector $B \in \mathscr{M}_{n}(\mathbb{K})$ can be chosen as the matrix of a bi-linear mapping satisfying (21). To sum up, we can formulate the following result as the answer to the problem of existence of a non-identically zero bi-additive mapping satisfying (19).

Theorem 6. Let $\mathbb{K}=\mathrm{GF}(q)$, where $q=p^{n}$ for some prime number $p$ and $n \in \mathbb{N}$. Consider the polynomial

$$
P(u, v, w)=\operatorname{det}\left(P_{u, v}-w \cdot \mathrm{id}\right) \quad(u, v, w \in \mathbb{K}),
$$

where id stands for the identity mapping of the linear space of matrices of order $n$ over the field $\mathbb{K}$. Then the following assertions are equivalent

(i) there is a not identically zero bi-additive mapping satisfying the semihomogeneity Eq. (21),

(ii) the characteristic polynomial of the linear operator $P_{\alpha, \beta}$ is reducible over the field $\mathbb{K}$ by one of its non-zero roots,

(iii) $P(\alpha, \beta, \gamma)=0$. 
Remark. If the elements $\alpha$ and $\beta$ are given, then the possible $\gamma$ 's are among the roots of the characteristic polynomial $P_{\alpha, \beta}$. The characteristic polynomial is independent of the choice of the basis $b_{0}, \ldots, b_{n-1}$. Moreover it is a polynomial over the prime field but the root $\gamma$ belongs to $\mathbb{K}$ in general. Setting the variables $\alpha$ and $\beta$ free, the roots of the multivariate polynomial $P$ is independent of the choice of the basis $b_{0}, \ldots, b_{n-1}$. In other words the algebraic variety

$$
P(x, y, z)=0
$$

in $\mathbb{K}^{3}$ contains all possible triplets for the solution of the semi-homogeneity Eq. (19).

\subsection{An example: the field GF(4)}

The operations are summarized in the following tables:

\begin{tabular}{lllll}
\hline+ & 0 & 1 & $a$ & $1+a$ \\
\hline 0 & 0 & 1 & $a$ & $1+a$ \\
1 & 1 & 0 & $1+a$ & $a$ \\
$a$ & $a$ & $1+a$ & 0 & 1 \\
$1+a$ & $1+a$ & $a$ & 1 & 0 \\
\hline
\end{tabular}

and

\begin{tabular}{lllll}
\hline$\cdot$ & 0 & 1 & $a$ & $1+a$ \\
\hline 0 & 0 & 0 & 0 & 0 \\
1 & 0 & 1 & $a$ & $1+a$ \\
$a$ & 0 & $a$ & $1+a$ & 1 \\
$1+a$ & 0 & $1+a$ & 1 & $a$ \\
\hline
\end{tabular}

Since $4=2^{2}$ it follows that $\mathrm{GF}(4)$ is a two-dimensional linear space over its prime field $\mathbb{Z}_{2}$. The basis we are going to use in the following is $b_{0}=1, b_{1}=a$. An easy computation shows that the translations $\tau_{0}$ and $\tau_{1}$ are represented by the matrices

$$
M^{0}=\left(\begin{array}{ll}
1 & 0 \\
0 & 1
\end{array}\right) \quad \text { and } \quad M^{1}=\left(\begin{array}{ll}
0 & 1 \\
1 & 1
\end{array}\right),
$$

respectively. Choosing elements

$$
\alpha=\alpha_{0}+\alpha_{1} \cdot a, \quad \beta=\beta_{0}+\beta_{1} \cdot a,
$$

where $\alpha_{0}, \alpha_{1}, \beta_{0}, \beta_{1} \in \mathbb{Z}_{2}$, it follows that 


$$
\begin{aligned}
P_{\alpha, \beta}(X)= & \alpha_{0} \beta_{0} X+\alpha_{0} \beta_{1} X\left(\begin{array}{ll}
0 & 1 \\
1 & 1
\end{array}\right)+\alpha_{1} \beta_{0}\left(\begin{array}{ll}
0 & 1 \\
1 & 1
\end{array}\right) X \\
& +\alpha_{1} \beta_{1}\left(\begin{array}{ll}
0 & 1 \\
1 & 1
\end{array}\right) X\left(\begin{array}{ll}
0 & 1 \\
1 & 1
\end{array}\right),
\end{aligned}
$$

where $X=\left(\begin{array}{ll}x_{00} & x_{01} \\ x_{10} & x_{11}\end{array}\right)$. Taking

$$
P_{\alpha, \beta}(X)=\left(\begin{array}{ll}
y_{00} & y_{01} \\
y_{10} & y_{11}
\end{array}\right),
$$

a direct computation shows that

$$
\begin{aligned}
y_{00}= & \alpha_{0} \beta_{0} x_{00}+\alpha_{0} \beta_{1} x_{01}+\alpha_{1} \beta_{0} x_{10}+\alpha_{1} \beta_{1} x_{11}, \\
y_{01}= & \alpha_{0} \beta_{0} x_{01}+\alpha_{0} \beta_{1}\left(x_{00}+x_{01}\right)+\alpha_{1} \beta_{0} x_{11}+\alpha_{1} \beta_{1}\left(x_{10}+x_{11}\right), \\
y_{10}= & \alpha_{0} \beta_{0} x_{10}+\alpha_{0} \beta_{1} x_{11}+\alpha_{1} \beta_{0}\left(x_{00}+x_{10}\right)+\alpha_{1} \beta_{1}\left(x_{01}+x_{11}\right), \\
y_{11}= & \alpha_{0} \beta_{0} x_{11}+\alpha_{0} \beta_{1}\left(x_{10}+x_{11}\right) \\
& +\alpha_{1} \beta_{0}\left(x_{01}+x_{11}\right)+\alpha_{1} \beta_{1}\left(x_{00}+x_{01}+x_{10}+x_{11}\right) .
\end{aligned}
$$

Therefore $P_{\alpha, \beta}$ is represented by the matrix

$$
\left(\begin{array}{cccc}
\alpha_{0} \beta_{0} & \alpha_{0} \beta_{1} & \alpha_{1} \beta_{0} & \alpha_{1} \beta_{1} \\
\alpha_{0} \beta_{1} & \alpha_{0} \beta_{0}+\alpha_{0} \beta_{1} & \alpha_{1} \beta_{1} & \alpha_{1} \beta_{0}+\alpha_{1} \beta_{1} \\
\alpha_{1} \beta_{0} & \alpha_{1} \beta_{1} & \alpha_{0} \beta_{0}+\alpha_{1} \beta_{0} & \alpha_{0} \beta_{1}+\alpha_{1} \beta_{1} \\
\alpha_{1} \beta_{1} & \alpha_{1} \beta_{0}+\alpha_{1} \beta_{1} & \alpha_{0} \beta_{1}+\alpha_{1} \beta_{1} & \alpha_{0} \beta_{0}+\alpha_{0} \beta_{1}+\alpha_{1} \beta_{0}+\alpha_{1} \beta_{1}
\end{array}\right)
$$

with respect to the basis

$$
B_{00}=\left(\begin{array}{ll}
1 & 0 \\
0 & 0
\end{array}\right), \quad B_{01}=\left(\begin{array}{ll}
0 & 1 \\
0 & 0
\end{array}\right), \quad B_{10}=\left(\begin{array}{ll}
0 & 0 \\
1 & 0
\end{array}\right), \quad B_{11}=\left(\begin{array}{ll}
0 & 0 \\
0 & 1
\end{array}\right) .
$$

If $\alpha=1+a$, i.e. $\alpha_{0}=\alpha_{1}=1$ and $\beta=a$, i.e. $\beta_{0}=0, \beta_{1}=1$, then we have the matrix

$$
\left(\begin{array}{llll}
0 & 1 & 0 & 1 \\
1 & 1 & 1 & 1 \\
0 & 1 & 0 & 0 \\
1 & 1 & 0 & 0
\end{array}\right)
$$

and the characteristic polynomial is

$$
P_{1+a, a}(t)=(t-1)^{2} \cdot\left(1+t+t^{2}\right) .
$$

This means that the possible choices are $\gamma=1, a$ or $1+a$, that is, if $X$ and $Y$ are linear spaces over the field $\mathbb{K}=\mathrm{GF}(4)$, then there exist not identically zero bi-additive mappings of the form $A: X \times Y \rightarrow \mathbb{K}$ such that

$$
\begin{aligned}
& A((1+a) x, a y)=A(x, y) \quad(x \in X, y \in Y), \\
& A((1+a) x, a y)=a A(x, y) \quad(x \in X, y \in Y),
\end{aligned}
$$


or

$$
A((1+a) x, a y)=(1+a) A(x, y) \quad(x \in X, y \in Y) .
$$

Remark. GF(4) seems to be rich in semi-homogeneous biadditive functions in the case of $\alpha=1+a$ and $\beta=a$. In general, if $\alpha$ and $\beta$ are fixed, then the characteristic polynomial is of degree $n^{2}$, i.e. we have at most $n^{2}$ different possible values for $\lambda$. It is a polynomial dependence on $n$ but the number of elements in $\operatorname{GF}\left(p^{n}\right)$ increases exponentially. Therefore the probability of a randomly chosen element in $\mathbb{K}$ to be a possible value for $\lambda$ tends to zero.

In this last section of the paper we investigated the existence of non-zero, bi-additive semi-homogeneous mappings. If the field $\mathbb{K}$ is of characteristic zero or $\mathbb{K}$ is a finite field, we could provide necessary and sufficient conditions. At the same time, there exist infinite fields of prime characteristic (for example, the field of all rational functions over $\mathbb{Z} / p \mathbb{Z}$ ). Therefore, we end this paper with two open problems.

Open Problem 1. Let $p$ be a prime and $\mathbb{K}$ be an infinite field of characteristic $p$. Further, let $X$ be a linear space over $\mathbb{K}$ and $a: X \rightarrow \mathbb{K}$ be an additive function. Find necessary and sufficient conditions for $a$ to be a nontrivial, semi-homogeneous additive mapping.

Open Problem 2. Let $p$ be a prime and $\mathbb{K}$ be an infinite field of characteristic $p$. Further, let $X$ and $Y$ be linear spaces over $\mathbb{K}$ and $A: X \times Y \rightarrow \mathbb{K}$ be a bi-additive function. Find necessary and sufficient conditions for $A$ to be a nontrivial, semi-homogeneous bi-additive mapping.

\section{Acknowledgements}

Open access funding provided by University of Debrecen (DE).

Open Access. This article is distributed under the terms of the Creative Commons Attribution 4.0 International License (http://creativecommons.org/licenses/by/4.0/), which permits unrestricted use, distribution, and reproduction in any medium, provided you give appropriate credit to the original author(s) and the source, provide a link to the Creative Commons license, and indicate if changes were made.

Publisher's Note Springer Nature remains neutral with regard to jurisdictional claims in published maps and institutional affiliations.

\section{References}

[1] Aczél, J.: Lectures on functional equations and their applications. In: Oser, H. (ed.) Mathematics in Science and Engineering, vol. 19. Academic Press, New York (1966) (Translated by Scripta Technica, Inc. Supplemented by the author)

[2] Aczél, J., Haruki, H., McKiernan, M.A., Sakovič, G.N.: General and regular solutions of functional equations characterizing harmonic polynomials. Aequ. Math. 1, 37-53 (1968) 
[3] Bahyrycz, A., Olko, J.: Hyperstability of some functional equations on restricted domain. J. Funct. Spaces. Art. ID 1946394, 6 (2017)

[4] Bahyrycz, A., Olko, J.: On stability and hyperstability of an equation characterizing multi-Cauchy-Jensen mappings. Results Math. 73(2), 55 (2018)

[5] Chung, J.K., Ebanks, B.R., Ng, C.T., Sahoo, P.K., Zeng, W.B.: On generalized rectangular and rhombic functional equations. Publ. Math. Debr. 47(3-4), 249-270 (1995)

[6] Daróczy, Z.: Notwendige und hinreichende Bedingungen für die Existenz von nichtkonstanten Lösungen linearer Funktionalgleichungen. Acta Sci. Math. Szeged 22, 31-41 (1961)

[7] Kiss, G.: Linear functional equations. Ann. Univ. Sci. Bp. Eötvös Sect. Math. 58, 125$132(2015)$

[8] Kiss, G., Laczkovich, M.: Linear functional equations, differential operators and spectral synthesis. Aequ Math 89(2), 301-328 (2015)

[9] Kiss, G., Varga, A.: Existence of nontrivial solutions of linear functional equations. Aequ. Math. 88(1-2), 151-162 (2014)

[10] Kiss, G., Varga, A., Vincze, C.: Algebraic methods for the solution of linear functional equations. Acta Math. Hung. 146(1), 128-141 (2015)

[11] Kuczma, M.: An introduction to the theory of functional equations and inequalities. In: Cauchy's Equation and Jensen's Inequality, 2nd edn. Birkhäuser Verlag, Basel (2009)

[12] Laczkovich, M., Székelyhidi, G.: Harmonic analysis on discrete abelian groups. Proc. Am. Math. Soc. 133, 1581-1586 (2005)

[13] Lidl, R., Niederreiter, H.: Introduction to Finite Fields and Their Applications. Cambridge University Press, Cambridge (1994)

[14] Stetkær, H.: Generalized rectangular functional equations. Aequ. Math. 57(1), 4-20 (1999)

[15] Székelyhidi, L.: On a class of linear functional equations. Publ. Math. Debr. 29(1-2), 19-28 (1982)

[16] Székelyhidi, L.: On a linear functional equation. Aequ. Math. 38(2-3), 113-122 (1989)

[17] Székelyhidi, L.: Convolution Type Functional Equations on Topological Abelian Groups. World Scientific Publishing Co. Inc, Teaneck, NJ (1991)

[18] Varga, A., Vincze, C.: On Daróczy's problem for additive functions. Publ. Math. Debr. 75(1-2), 299-310 (2009)

[19] Varga, A., Vincze, C.: Nontrivial solutions of linear functional equations: methods and examples. Opusc. Math. 35(6), 957-972 (2015)

[20] Vincze, C., Varga, A.: On the characteristic polynomials of linear functional equations. Period. Math. Hungar. 71(2), 250-260 (2015)

Eszter Gselmann and Csaba Vincze

Institute of Mathematics

University of Debrecen

P.O. Box 400 Debrecen 4002

Hungary

e-mail: gselmann@science.unideb.hu

Csaba Vincze

e-mail: csvincze@science.unideb.hu 
Gergely Kiss

Alfréd Rényi Institute of Mathematics

Hungarian Academy of Science

Reáltanoda u. 13-15.

Budapest 1053

Hungary

e-mail: kigergo57@gmail.com

Received: March 19, 2019

Revised: August 15, 2019 